\title{
| | | \\ THE BATALIN-VILKOVISKY STRUCTURE OVER THE HOCHSCHILD COHOMOLOGY RING OF A GROUP ALGEBRA
}

\author{
YUMING LIU AND GUODONG ZHOU*
}

\begin{abstract}
We realize explicitly the well-known additive decomposition of the Hochschild cohomology ring of a group algebra in the elements level. As a result, we describe the cup product, the Batalin-Vilkovisky operator and the Lie bracket in the Hochschild cohomology ring of a group algebra.
\end{abstract}

\section{INTRODUCTION}

Let $k$ be a field and $G$ a finite group. Then the Hochschild cohomology ring of the group algebra $k G$ admits an additive decomposition:

$$
H H^{*}(k G) \simeq \bigoplus_{x \in X} H^{*}\left(C_{G}(x), k\right)
$$

where $X$ is a set of representatives of conjugacy classes of elements of $G$ and $C_{G}(x)$ is the centralizer of $x \in G$. The proof of this isomorphism can be found in [2] or [16. The usual proof is abstract rather than giving an explicit isomorphism. For example, one of the key steps is to use the so-called Eckmann-Shapiro Lemma, one need to construct some comparison maps between two projective resolutions in order to write it down explicitly, and this is usually difficult. In [16, Siegel and Witherspoon used techniques and notations from group representation theory to interpret the above additive decomposition explicitly. For our purpose, we need to give an explicit isomorphism in the elements level.

A priori, the additive decomposition gives an isomorphism of graded vector spaces. The left handed side has a graded commutative algebra structure given by the cup product, a graded Lie algebra structure given by the Gerstenhaber Lie bracket (8), and a Batalin-Vilkovisky (BV) algebra structure given by the $\triangle$ operator $([18])$. It would be interesting to describe these structures in terms of pieces from the right handed side.

For graded algebra structure, it was done by Holm for abelian groups using computations ([10]), then Cibils and Solotar gave a conceptual proof in (4 $)$. The general case was dealt with by Siegel and Witherspoon ([16]), they described the cup product formula by notations from group representation theory. Our goal in the present paper is to represent the cup product, the Lie bracket and the BV operator in the Hochschild cohomology ring in terms of the additive decomposition. This is based on the explicit construction of an isomorphism in the additive decomposition (although there is no canonical choice for such an isomorphism).

The main obstruction in realizing an isomorphism in the additive decomposition comes from the fact that, it is usually difficult to construct the comparison map between two projective resolutions of modules. There is a surprising way to simplify such construction, namely, one can reduce it to construct a setwise self-homotopy over one projective resolution, which is often much easier. This method was already used in a recent paper by the second author jointly with Le (11]). For convenience, we shall give a brief introduction to this idea in Section 2.

* Corresponding author.

Mathematics Subject Classification(2010): 16E40, 18G10, $20 \mathrm{C05.}$

Keywords: Additive decomposition; Batalin-Vilkovisky structure; Cup product; Group cohomology; Hochschild cohomology ring; Normalized bar resolution; Setwise self-homotopy.

Date: version of October 8, 2018.

Both authors are supported by the exchange program STIC-Asie 'ESCAP' financed by the French Ministry of Foreign Affairs. The first author is supported by NCET Program from MOE of China and by NNSF (No.11171325, No.11331006, No.61070251). The second author is supported by by Shanghai Pujiang Program (No.13PJ1402800), by National Natural Science Foundation of China (No.11301186) and by the Doctoral Fund of Youth Scholars of Ministry of Education of China (No.20130076120001). 
This article is organized as follows. In Section 2, we recall Le and Zhou's method on constructing comparison maps. In Section 3 and 4, we review the definitions of various structures over Hochschild cohomology and group cohomology, using the normalized bar resolutions. We always use the normalized bar resolutions since they are easy to describe and can greatly simplify the computations.

In Section 5, we give a way to realize explicitly the additive decomposition of the Hochschild cohomology of a group algebra. The main line of our method follows from [16]. In Section 6, we shall use some idea from [4] to give another way to realize the additive decomposition.

We give the cup product formula in Section 7. Our formula shows that the group cohomology $H^{*}(G, k)$ can be seen as a subalgebra of the Hochschild cohomology $H H^{*}(k G)$ in the complex level, and that the additive decomposition naturally gives an isomorphism of graded $H^{*}(G, k)$-modules.

We deal with the $\triangle$ operator and the graded Lie bracket in the next section. In particular, we show that the operator $\triangle$ restricts to each summand under the additive decomposition, and that $H^{*}(G, k)$ is indeed a BV subalgebra of $H H^{*}(k G)$.

In the final section, we use our formulae to compute the BV structure of the Hochschild cohomology ring for symmetric group of degree 3 over $\mathbb{F}_{3}$. To the best of our knowledge, this is the first concrete computation on the BV structure of a non-commutative algebra.

\section{How to construct comparison morphisms? [11, Appendix]}

Definition 2.1. (cf. [1]) Let $A$ be an algebra over a field $k$. Let

$$
C^{*}: \cdots \longrightarrow C_{n+1} \stackrel{d_{n+1}}{\longrightarrow} C_{n} \stackrel{d_{n}}{\longrightarrow} C_{n-1} \longrightarrow \cdots
$$

be a chain complex of $A$-modules. If there are maps (just as maps between sets) $s_{n}: C_{n} \longrightarrow C_{n+1}$ such that $s_{n-1} d_{n}+d_{n+1} s_{n}=i d_{C_{n}}$ for all $n$, then the maps $\left\{s_{n}\right\}$ are called a setwise self-homotopy over the complex $C^{*}$.

Remark 2.2. $\quad$ (i) There is a setwise self-homotopy over a complex $C^{*}$ of $A$-modules if and only if $C^{*}$ is an exact complex, that is, $C^{*}$ is a zero object in the derived category $\operatorname{D}(\operatorname{Mod} A)$. Compare this with the usual self-homotopy, which is equivalent to saying that $C^{*}$ is split exact, and hence it is a zero object in the homotopy category $\operatorname{K}(\operatorname{Mod} A)$.

(ii) Usually a setwise self-homotopy can be taken to be linear maps, so it is a self-homotopy in the usual sense in the category of complexes of $k$-vector spaces. In case that the exact complex is a right bounded complex of $A$ - $A$-bimodules, a setwise self-homotopy can even be chosen as homomorphisms of one-sided modules.

We will show how to use a setwise self-homotopy to construct a comparison map. Let $M$ and $N$ be two $A$-modules, and let $f: M \longrightarrow N$ be an $A$-module homomorphism. Suppose that $P^{*}=\left(P_{i}, \partial_{i}\right)$ is a free resolution of $M$, and that $Q^{*}=\left(Q_{i}, d_{i}\right)$ is a projective resolution of $N$. Suppose further that there is a setwise self-homotopy $s=\left\{s_{n}\right\}$ over $Q^{*}$ (including $N$ ):

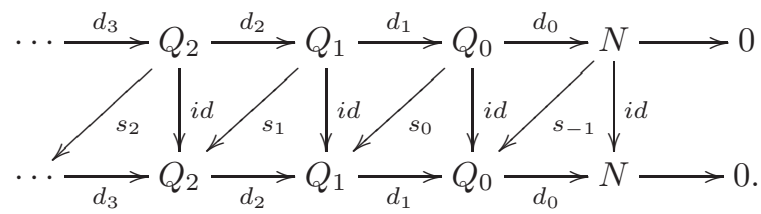

For each $i \geq 0$, choose a basis $X_{i}$ for the free $A$-module $P_{i}$ (the $i$-th term of $P^{*}$ ). We define inductively the maps $f_{i}: X_{i} \longrightarrow Q_{i}$ as follows: for $x \in X_{0}, f_{0}(x)=s_{-1} f \partial_{0}(x)$; for $i>1$ and for $x \in X_{i}, f_{i}(x)=s_{i-1} f_{i-1} \partial_{i}(x)$. Extending $A$-linearly the maps $f_{i}$ we get $A$-homomorphisms $f_{i}: P_{i} \longrightarrow Q_{i}$. It is easy to verify that $\left\{f_{i}\right\}$ gives a chain map between the complexes $P^{*}$ and $Q^{*}$. We illustrate the above procedure in the following diagram: 

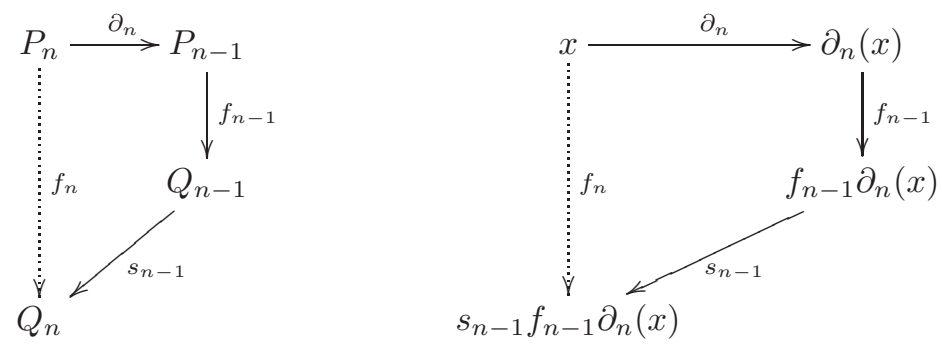

We shall use the following standard homological fact.

Lemma 2.3. Let $A$ and $B$ be two rings and let $F: M o d A \longrightarrow M o d B$ be an additive contravariant (resp., covariant) functor. If $C^{*}$ and $D^{*}$ are two projective resolutions of an $A$-module $M$, then the cochain complexes $F C^{*}$ and $F D^{*}$ of $B$-modules are homotopic. In particular, if $\varphi: C^{*} \longrightarrow D^{*}$ and $\psi: D^{*} \longrightarrow C^{*}$ are two chain maps inducing identity maps $i d_{M}: M \longrightarrow M$, then $F \varphi: F D^{*} \longrightarrow F C^{*}$ (resp., $\left.F \varphi: F C^{*} \longrightarrow F D^{*}\right)$ and $F \psi: F C^{*} \longrightarrow F D^{*}$ (resp., $F \psi: F D^{*} \longrightarrow F C^{*}$ ) are inverse homotopy equivalences.

\section{Remainder on Hochschild COHOMOLOGY}

In this section, we recall the definitions of various structures over Hochschild cohomology. For the cup product and the Lie bracket in the Hochschild cohomology ring, we refer to Gerstenhaber's original paper [8; for the Batalin-Vilkovisky algebra structure, we refer to Tradler [18].

Let $k$ be a field and $A$ an associative $k$-algebra with identity $1_{A}$. Denote by $\bar{A}$ the quotient space $A /\left(k \cdot 1_{A}\right)$. We shall write $\otimes$ for $\otimes_{k}$ and $A^{\otimes n}$ for the $n$-fold tensor product $A \otimes \cdots \otimes A$. The normalized bar resolution $\left(\operatorname{Bar}_{*}(A), d_{*}\right)$ of $A$ is a free resolution of $A$ as $A$-A-bimodules, where

$$
\operatorname{Bar}_{-1}(A)=A, \text { and for } n \geq 0, \quad \operatorname{Bar}_{n}(A)=A \otimes \bar{A}^{\otimes n} \otimes A,
$$

$d_{0}: \operatorname{Bar}_{0}(A)=A \otimes A \longrightarrow A, \quad a_{0} \otimes a_{1} \longmapsto a_{0} a_{1}$ (multiplication map), and for $n \geq 1$,

$d_{n}: \operatorname{Bar}_{n}(A) \longrightarrow \operatorname{Bar}_{n-1}(A), \quad a_{0} \otimes \overline{a_{1}} \otimes \cdots \otimes \overline{a_{n}} \otimes a_{n+1} \longmapsto \sum_{i=0}^{n}(-1)^{i} a_{0} \otimes \cdots \otimes \overline{a_{i} a_{i+1}} \otimes \cdots \otimes a_{n+1}$.

The normalized bar resolution is a natural quotient complex of the usual bar resolution. The exactness of the normalized bar resolution is an easy consequence of the following fact: there is a setwise self-homotopy $s_{n}: \operatorname{Bar}_{n}(A) \longrightarrow \operatorname{Bar}_{n+1}(A)$ over $\operatorname{Bar}_{*}(A)$ given by

$$
s_{n}\left(a_{0} \otimes \overline{a_{1}} \otimes \cdots \otimes \overline{a_{n}} \otimes a_{n+1}\right)=1 \otimes a_{0} \otimes \overline{a_{1}} \otimes \cdots \otimes \overline{a_{n}} \otimes a_{n+1} .
$$

Notice that here each $s_{n}$ is just a right $A$-module homomorphism. For simplicity, in the following we will write $a_{i}$ for $\overline{a_{i}}$.

Let ${ }_{A} M_{A}$ be an $A$ - $A$-bimodule. Remember that any $A$ - $A$-bimodule can be identified with a left module over the enveloping algebra $A^{e}=A \otimes A^{o p}$. We have the Hochschild cohomology complex $\left(C^{*}(A, M), \delta_{*}\right)$ :

$$
\begin{gathered}
C^{n}(A, M)=\operatorname{Hom}_{A^{e}}\left(\operatorname{Bar}_{n}(A), M\right) \simeq \operatorname{Hom}_{k}\left(\bar{A}^{\otimes n}, M\right), \quad \text { for } n \geq 0, \\
\delta_{n}: C^{n}(A, M) \longrightarrow C^{n+1}(A, M), \quad f \longmapsto \delta_{n}(f), \quad \text { where } \delta_{n}(f) \text { sends } a_{1} \otimes \cdots \otimes a_{n+1} \text { to } \\
a_{1} f\left(a_{2} \otimes \cdots \otimes a_{n+1}\right)+\sum_{i=1}^{n}(-1)^{i} f\left(a_{1} \otimes \cdots \otimes a_{i} a_{i+1} \otimes \cdots \otimes a_{n+1}\right)+(-1)^{n+1} f\left(a_{1} \otimes \cdots \otimes a_{n}\right) a_{n+1} .
\end{gathered}
$$

For $n \geq 0$, the degree- $n$ Hochschild cohomology group of the algebra $A$ with coefficients in $M$ is defined to be

$$
H H^{n}(A, M)=H^{n}\left(C^{*}(A, M)\right) \simeq \operatorname{Ext}_{A^{e}}^{n}(A, M) .
$$

If in particular, $A=k G$ the group algebra of a finite group $G$, then the Hochschild cohomology complex $\left(C^{*}(A, M), \delta_{*}\right)$ has the following form:

$$
C^{n}(k G, M) \simeq \operatorname{Hom}_{k}\left(\overline{k G}^{\otimes n}, M\right) \simeq \operatorname{Map}\left(\bar{G}^{n}, M\right), \text { for } n \geq 0,
$$


where $\bar{G}=G-\{1\}$ and $M a p\left(\bar{G}^{\times n}, M\right)$ denotes all the maps between the sets $\bar{G}^{\times n}$ and $M$, and the differential is given by

$$
\delta_{n}: \operatorname{Map}\left(\bar{G}^{\times n}, M\right) \longrightarrow \operatorname{Map}\left(\bar{G}^{\times n+1}, M\right), \quad f \longmapsto \delta_{n}(f),
$$

where $\delta_{n}(f)$ sends $\left(g_{1}, \cdots, g_{n+1}\right) \in \bar{G}^{n+1}$ to

$$
g_{1} f\left(g_{2}, \cdots, g_{n+1}\right)+\sum_{i=1}^{n}(-1)^{i} f\left(g_{1}, \cdots, g_{i} g_{i+1}, \cdots, g_{n+1}\right)+(-1)^{n+1} f\left(g_{1}, \cdots, g_{n}\right) g_{n+1} .
$$

When $M=A$ with the obvious $A$-A-bimodule structure, we write $C^{n}(A)$ (resp. $\left.H H^{n}(A)\right)$ for $C^{n}(A, A)$ (resp. $\left.H H^{n}(A, A)\right)$. Let $f \in C^{n}(A), g \in C^{m}(A)$. Then the cup product $f \cup g \in C^{n+m}(A)$ is defined as follows:

$$
f \cup g: \bar{A}^{\otimes(n+m)} \longrightarrow A, \quad a_{1} \otimes \cdots \otimes a_{n+m} \longmapsto f\left(a_{1} \otimes \cdots \otimes a_{n}\right) g\left(a_{n+1} \otimes \cdots \otimes a_{n+m}\right) .
$$

This cup product is associative and induces a well-defined product over

$$
H H^{*}(A)=\bigoplus_{n \geq 0} H H^{n}(A)=\bigoplus_{n \geq 0} \operatorname{Ext}_{A^{e}}^{n}(A, A),
$$

which is called the Hochschild cohomology ring of $A$. Moreover, $H H^{*}(A)$ is graded commutative, that is, $\alpha \cup \beta=(-1)^{m n} \beta \cup \alpha$ for $\alpha \in H H^{n}(A)$ and $\beta \in H H^{m}(A)$. As usual, we call an element $\alpha \in H H^{n}(A)$ homogeneous of degree $n$, and its degree will be denoted by $|\alpha|$.

The Lie bracket is defined as follows. Let $f \in C^{n}(A, M), g \in C^{m}(A)$. If $n, m \geq 1$, then for $1 \leq i \leq n$, the so-called brace operation $f \circ_{i} g \in C^{n+m-1}(A, M)$ is defined by

$$
f \circ_{i} g\left(a_{1} \otimes \cdots a_{n+m-1}\right)=f\left(a_{1} \otimes \cdots \otimes a_{i-1} \otimes g\left(a_{i} \otimes \cdots \otimes a_{i+m-1}\right) \otimes a_{i+m} \otimes \cdots \otimes a_{n+m-1}\right) ;
$$

if $n \geq 1$ and $m=0$, then $g \in A$ and for $1 \leq i \leq n$, set

$$
f \circ_{i} g\left(a_{1} \otimes \cdots a_{n-1}\right)=f\left(a_{1} \otimes \cdots \otimes a_{i-1} \otimes g \otimes a_{i} \otimes \cdots \otimes a_{n-1}\right) ;
$$

for any other case, set $f \circ_{i} g$ to be zero. Define

$$
f \circ g=\sum_{i=1}^{n}(-1)^{(m-1)(i-1)} f \circ_{i} g \in C^{n+m-1}(A, M)
$$

and for $f \in C^{n}(A), g \in C^{m}(A)$, define

$$
[f, g]=f \circ g-(-1)^{(n-1)(m-1)} g \circ f \in C^{n+m-1}(A) .
$$

The above [, ] induces a well-defined (graded) Lie bracket in Hochschild cohomology

$$
[,]: H H^{n}(A) \times H H^{m}(A) \longrightarrow H H^{n+m-1}(A)
$$

such that $\left(H H^{*}(A), \cup,[],\right)$ is a Gerstenhaber algebra, that is, for homogeneous elements $\alpha, \beta, \gamma$ in $H H^{*}(A)$, the following three conditions hold:

- $\left(H H^{*}(A), \cup\right)$ is an associative algebra and it is graded commutative, that is, the cup product $\cup$ is an associative multiplication and satisfies $\alpha \cup \beta=(-1)^{|\alpha||\beta|} \beta \cup \alpha$;

- $\left(H H^{*}(A),[],\right)$ is a graded Lie algebra, that is, the Lie bracket [, ] satisfies $[\alpha, \beta]=$ $-(-1)^{(|\alpha|-1)(|\beta|-1)}[\beta, \alpha]$ and the graded Jacobi identity;

- Possion rule: $[\alpha \cup \beta, \gamma]=[\alpha, \gamma] \cup \beta+(-1)^{|\alpha|(|\gamma|-1)} \alpha \cup[\beta, \gamma]$.

We now assume that $A$ is a symmetric $k$-algebra, that is, $A$ is isomorphic to its dual $D(A)=$ $\operatorname{Hom}_{k}(A, k)$ as $A^{e}$-modules, or equivalently, if there exists a symmetric associative non-degenerate bilinear form $\langle\rangle:, A \times A \longrightarrow k$. This bilinear form induces a duality between the Hochschild cohomology and the Hochschild homology. In fact, for any $n \geq 0$ there is an isomorphism between $H H^{n}(A)$ and $H H_{n}(A)$ induced by the following canonical isomorphisms

$$
\operatorname{Hom}_{k}\left(A \otimes A^{e} \operatorname{Bar}_{n}(A), k\right) \simeq \operatorname{Hom}_{A^{e}}\left(\operatorname{Bar}_{n}(A), D(A)\right) \simeq \operatorname{Hom}_{A^{e}}\left(\operatorname{Bar}_{n}(A), A\right)
$$


Via this duality, we have, for $n \geq 1$, an operator $\triangle: C^{n}(A) \longrightarrow C^{n-1}(A)$ which corresponds to the Connes' $B$-operator (denoted by $B$ ) on the Hochschild homology complex. More precisely, for any $f \in C^{n}(A), \triangle(f) \in C^{n-1}(A)$ is given by the equation

$$
\left\langle\triangle(f)\left(a_{1} \otimes \cdots \otimes a_{n-1}\right), a_{n}\right\rangle=\sum_{i=1}^{n}(-1)^{i(n-1)}\left\langle f\left(a_{i} \otimes \cdots \otimes a_{n-1} \otimes a_{n} \otimes a_{1} \otimes \cdots \otimes a_{i-1}\right), 1\right\rangle .
$$

From the well-known properties of the Connes' $B$-operator $B$ (cf. [12, Chapter 2]), it is easy to see that the operator $\triangle$ is a chain map such that the induced operation $\triangle$ on Hochschild cohomology $H H^{*}(A)$ squares to zero (in fact, $\triangle^{2}=0$ holds on normalized Hochschild cochain complex level). It turns out that the Gerstenhaber algebra $\left(H H^{*}(A), \cup,[],\right)$ together with the operator $\triangle$ is a BatalinVilkovsky algebra (BV-algebra), that is, in addition to be a Gerstenhaber algebra, $\left(H H^{*}(A), \triangle\right)$ is a complex and

$$
[\alpha, \beta]=-(-1)^{(|\alpha|-1)|\beta|}\left(\triangle(\alpha \cup \beta)-\triangle(\alpha) \cup \beta-(-1)^{|\alpha|} \alpha \cup \triangle(\beta)\right)
$$

for all homogeneous elements $\alpha, \beta \in H H^{*}(A)$.

Remark 3.1. The sign in the definition of a BV-algebra depends on the choice of the definitions of cup product and Lie bracket. If we define $\alpha \cup^{\prime} \beta=(-1)^{|\alpha||\beta|} \alpha \cup \beta$ and $\triangle^{\prime}(\alpha)=(-1)^{(|\alpha|-1)} \triangle(\alpha)$, then we get

$$
[\alpha, \beta]=(-1)^{|\alpha|}\left(\triangle^{\prime}\left(\alpha \cup^{\prime} \beta\right)-\triangle^{\prime}(\alpha) \cup^{\prime} \beta-(-1)^{|\alpha|} \alpha \cup^{\prime} \triangle^{\prime}(\beta)\right),
$$

which is the equality in the usual definition of a BV-algebra (see, for example [9, 14]). We choose the sign convention from [18] because of our convention of the definitions of cup product and Connes' $B$-operator in the Hochschild (co)homology theory.

\section{Remainder on GROUP COHOMOLOGY}

Let $G$ be a finite group and $U$ a left $k G$-module. The group cohomology of $G$ with coefficient in $U$ is defined to be $H^{n}(G, U)=\operatorname{Ext}_{k G}^{n}(k, U)$. The complex $\operatorname{Bar}_{*}(k G) \otimes_{k G} k$ is the standard resolution of the trivial module $k$. In fact, as the setwise self-homotopy $s_{n}$ over $\operatorname{Bar}_{*}(k G)$ are right module homomorphisms, $\operatorname{Bar}_{*}(k G) \otimes_{k G} k$ is exact and thus a projective resolution of $k G \otimes_{k} k \simeq k$. We write the complex $C^{*}(G, U)=\operatorname{Hom}_{k G}\left(\operatorname{Bar}_{*}(k G) \otimes_{k G} k, U\right)$. Therefore, for $n \geq 0$,

$$
\begin{aligned}
C^{n}(G, U) & \simeq \operatorname{Hom}_{k G}\left(\left(k G \otimes \overline{k G}^{\otimes n} \otimes k G\right) \otimes_{k G} k, U\right) \simeq \operatorname{Hom}_{k G}\left(k G \otimes \overline{k G}^{\otimes n}, U\right) \\
& \simeq \operatorname{Hom}_{k}\left(\overline{k G}^{\otimes n}, U\right) \simeq \operatorname{Map}\left(\bar{G}^{\times n}, U\right),
\end{aligned}
$$

and the differential is given by

$$
\delta_{0}(x)(g)=g x-x \quad(\text { for } x \in U \text { and } g \in \bar{G})
$$

and (for $\varphi: \bar{G}^{\times n} \longrightarrow U$ and $g_{1}, \cdots, g_{n+1} \in \bar{G}$ )

$$
\begin{aligned}
\delta_{n}(\varphi)\left(g_{1}, \cdots, g_{n+1}\right)=g_{1} \varphi\left(g_{2}, \cdots, g_{n+1}\right)+ \\
\qquad \sum_{i=1}^{n}(-1)^{i} \varphi\left(g_{1}, \cdots, g_{i} g_{i+1}, \cdots, g_{n+1}\right)+(-1)^{n+1} \varphi\left(g_{1}, \cdots, g_{n}\right) .
\end{aligned}
$$

Of particular interest to us are the following two cases which relate group cohomology to Hochschild cohomology and in fact which underly our two realisations of the additive decomposition of the Hochschild cohomology of a group algebra.

Note that we have an algebra isomorphism $(k G)^{e} \simeq k(G \times G)$ given by $g_{1} \otimes g_{2} \longmapsto\left(g_{1}, g_{2}^{-1}\right)$, for $g_{1}, g_{2} \in G$. Thus we can also identify each $k G$ - $k G$-bimodule $M$ as a left $k(G \times G)$-module by $\left(g_{1}, g_{2}\right) \cdot x=g_{1} x g_{2}^{-1}$. In the sequel, we shall write the Hochschild cohomology complex for the group algebra $k G$ in terms of $k(G \times G)$-modules.

Case 1. $M=k G$, the module $k G$ with the obvious $k G$ - $k G$-bimodule, or equivalently, the $k(G \times G)$-module $k G$ with action: $\left(g_{1}, g_{2}\right) \cdot x=g_{1} x g_{2}^{-1}$ for $g_{1}, g_{2} \in G$. Consider $G$ as a subgroup of $G \times G$ via the diagonal embedding $G \rightarrow G \times G, g \mapsto(g, g)$, and it is easy to verify that there is 
a $k(G \times G)$-module isomorphism $\operatorname{Ind} d_{G}^{G \times G} k=k(G \times G) \otimes_{k G} k \simeq k G,\left(g_{1}, g_{2}\right) \otimes 1 \longmapsto g_{1} g_{2}^{-1}$. So we have

$$
\begin{aligned}
H H^{n}(k G, k G) & \simeq \operatorname{Ext}_{k(G \times G)}^{n}(k G, k G) \simeq \operatorname{Ext}_{k(G \times G)}^{n}\left(\operatorname{Ind}_{G}^{G \times G} k, k G\right) \\
& \simeq \operatorname{Ext}_{k G}^{n}\left(k, \operatorname{Res}_{G}^{G \times G} k G\right)=\operatorname{Ext}_{k G}^{n}\left(k,{ }_{c} k G\right) \\
& =H^{n}\left(G,{ }_{c} k G\right),
\end{aligned}
$$

where the third isomorphism is given by the adjoint equivalence and ${ }_{c} k G$ is considered as a left $k G$-module by conjugation: $g \cdot x=g x g^{-1}$ for $g, x \in G$. This verifies a well-known fact observed by Eilenberg and Mac Lane ([5): the Hochschild cohomology $H H^{n}(k G, k G)$ of $k G$ with coefficients in $k G$ is isomorphic to the ordinary group cohomology $H^{n}(G, k G)$ of $G$ with coefficients in $k G$ under the conjugation.

Case 2. $M=k$, the trivial $k G$ - $k G$-bimodule, or equivalently, the $k(G \times G)$-module $k$ with action: $\left(g_{1}, g_{2}\right) \cdot 1=1$ for $g_{1}, g_{2} \in G$. Since we have

$$
H H^{n}(k G, k) \simeq \operatorname{Ext}_{k(G \times G)}^{n}(k G, k) \simeq \operatorname{Ext}_{k(G \times G)}^{n}\left(k(G \times G) \otimes_{k G} k, k\right) \simeq \operatorname{Ext}_{k G}^{n}(k, k)=H^{n}(G, k),
$$

the Hochschild cohomology $H H^{n}(k G, k)$ of $k G$ with coefficients in $k$ is isomorphic to the ordinary group cohomology $H^{n}(G, k)$. Another way to see this lies in the fact that the two complexes $C^{*}(k G, k)$ and $C^{*}(G, k)$ coincide.

We can deduce the second case from the first one. In fact, the subspace $k\left(\sum_{g \in G} g\right) \subseteq k G$ is a sub- $(G \times G)$-module of $k G$ (and also sub- $G$-module of ${ }_{c} k G$ ), which is isomorphic to the trivial module. Via the isomorphisms in Case $1, H H^{*}\left(k G, k\left(\sum_{g \in G} g\right)\right)$ corresponds to $H^{*}\left(G, k\left(\sum_{g \in G} g\right)\right)$.

We can in fact define a cup product and Lie bracket over $H^{*}(G, k)=\bigoplus_{n \geq 0} H^{n}(G, k)$ such that it becomes a Gerstenhaber algebra. One sees that the cup product and the Lie bracket over $H H^{*}(k G)$ restrict to $H^{*}(G, k)$ by [7. Corollary 2.2], so $H^{*}(G, k)$ is a Gerstenhaber subalgebra of $H H^{*}(k G)$. In fact, as in [7, Proof of Theorem 1.8], there is a chain map at the cohomology complex level:

$$
\begin{gathered}
\operatorname{Hom}_{k G}\left(\operatorname{Bar}_{n}(k G) \otimes_{k G} k, k\right)=C^{n}(k G, k) \hookrightarrow C^{n}(k G)=H_{k o m}(G \times G) \\
\left(\varphi: \bar{G}^{\times n} \longrightarrow k\right) \longmapsto\left(\psi: \bar{G}^{\times n}(k G), k G\right), \\
\longrightarrow k G), \quad \psi\left(g_{1}, \cdots, g_{n}\right)=\varphi\left(g_{1}, \cdots, g_{n}\right) g_{1} \cdots g_{n} .
\end{gathered}
$$

This inclusion map preserves the brace operations in the following sense:

Let $\varphi_{1} \in C^{n}(k G, k) \simeq \operatorname{Map}\left(\bar{G}^{\times n}, k\right), \varphi_{2} \in C^{m}(k G, k)$, and let $\widehat{\varphi}_{1} \in C^{n}(k G), \widehat{\varphi}_{2} \in C^{m}(k G)$ be the corresponding elements under the above inclusion map. Then $\widehat{\varphi}_{1} \circ_{i} \widehat{\varphi}_{2}=\widehat{\varphi_{1} \circ_{i} \widehat{\varphi}_{2}} \in C^{m+n-1}(k G)$.

Recall that $k G$ is a symmetric algebra with the bilinear form

$$
\begin{gathered}
\langle,\rangle: k G \times k G \longrightarrow k, \\
\langle g, h\rangle= \begin{cases}1 & \text { if } g=h^{-1} \\
0 & \text { otherwise }\end{cases}
\end{gathered}
$$

for $g, h \in G$. So there is a well-defined BV-algebra structure on $H H^{*}(k G)$. We shall see later that $H^{*}(G, k)$ is furthermore a sub-BV-algebra of $H H^{*}(k G)$.

\section{The FIRST REALIZATION OF THE ADDitive DECOMPOSITION}

Let $k$ be a field and $G$ a finite group. Then the Hochschild cohomology ring of the group algebra $k G$ admits an additive decomposition:

$$
H H^{*}(k G) \simeq \bigoplus_{x \in X} H^{*}\left(C_{G}(x), k\right)
$$

where $X$ is a set of representatives of conjugacy classes of elements of $G$ and $C_{G}(x)=\{g \in G \mid g x=$ $x g\}$ is the centralizer subgroup of $G$. In this section, we give an explicit construction of the additive decomposition. The main technique we used here is to construct comparison maps based on some setwise self-homotopys. 
The following is a proof of the additive decomposition which consists of a series of isomorphisms. Our first realization of the additive decomposition will follow this series of isomorphisms.

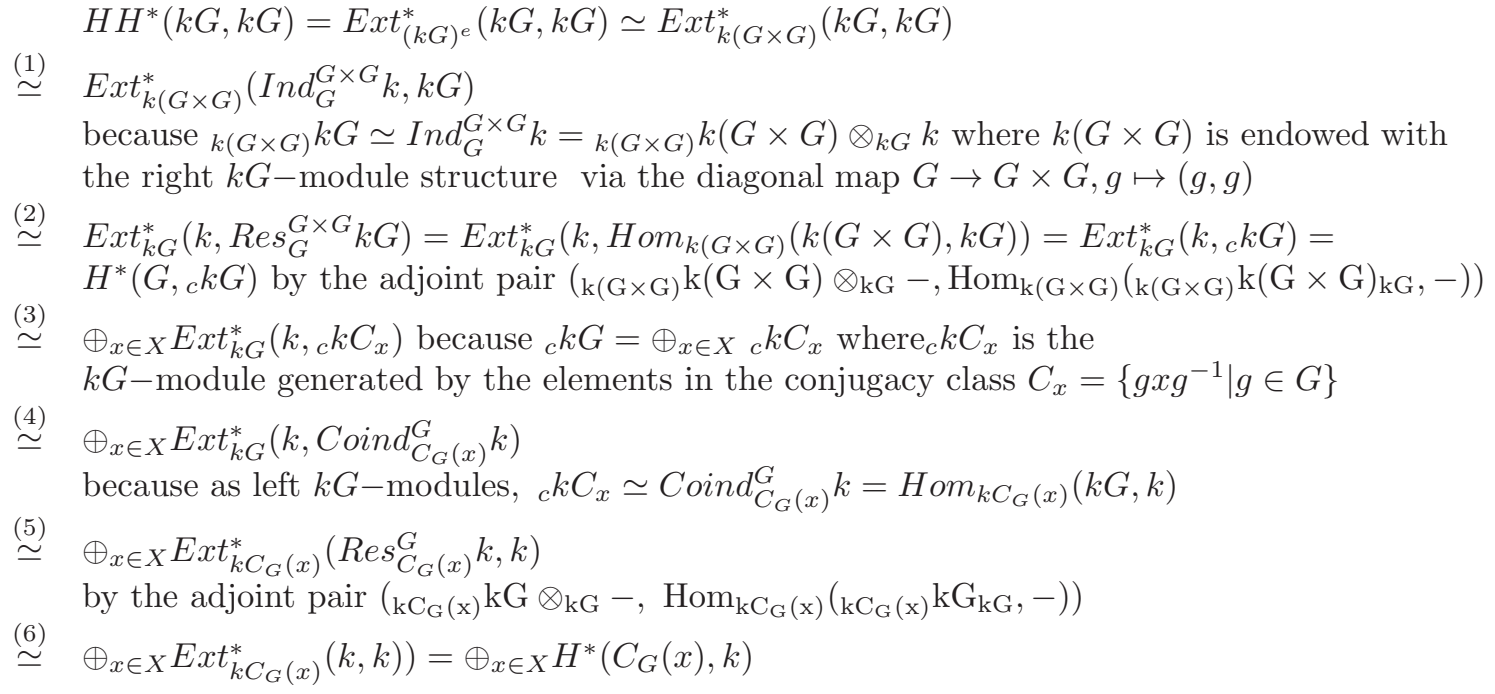

We shall express explicitly these isomorphisms step by step using the bar resolution.

The first step. By definition, the Hochschild cohomology groups $H H^{*}(k G, k G)$ can be computed using the bar resolution $\operatorname{Bar}_{*}(k G)$. On the other hand, $\operatorname{Bar}_{*}(k G) \otimes_{k G} k$ is a free resolution of $k$ as left $k G$-module and therefore, $k(G \times G) \otimes_{k G} \operatorname{Bar}_{*}(k G) \otimes_{k G} k$ is also a free resolution of the $k(G \times G)$-module $k(G \times G) \otimes_{k G} k \simeq k G$. Notice that the terms in $\operatorname{Bar}_{*}(k G)$ are still viewed as the usual $k G$ - $k G$-bimodules when we do the above tensor products.

Let us write explicitly the resolution $k(G \times G) \otimes_{k G} \operatorname{Bar}_{*}(k G) \otimes_{k G} k$. Under the identification

$$
\begin{aligned}
k(G \times G) \otimes_{k G} \operatorname{Bar}_{n}(k G) \otimes_{k G} k & \simeq k(G \times G) \otimes_{k G}\left(k G \otimes \overline{k G}^{\otimes n} \otimes k G\right) \otimes_{k G} k \\
& \simeq k(G \times G) \otimes \overline{k G}^{\otimes n} \\
& \simeq k G \otimes k G \otimes \overline{k G}^{\otimes n}
\end{aligned}
$$

and the differential is as follows (we only write down the maps on base elements here and later):

$$
\begin{gathered}
k G \otimes k G \longrightarrow k G, \quad x \otimes y \longmapsto x y^{-1} ; \\
k G \otimes k G \otimes \overline{k G} \longrightarrow k G \otimes k G, \quad x \otimes y \otimes g_{1} \longmapsto x g_{1} \otimes y g_{1}-x \otimes y ; \\
\ldots \ldots \otimes \\
k G \otimes k G \otimes \overline{k G}^{\otimes n} \longrightarrow k G \otimes k G \otimes \overline{k G}^{\otimes n-1}, \quad x \otimes y \otimes g_{1} \otimes \cdots \otimes g_{n} \longmapsto \\
x g_{1} \otimes y g_{1} \otimes g_{2} \otimes \cdots \otimes g_{n}+\sum_{i=1}^{n-1}(-1)^{i} x \otimes y \otimes g_{1} \otimes \cdots \otimes g_{i} g_{i+1} \otimes \cdots \otimes g_{n}+(-1)^{n} x \otimes y \otimes g_{1} \otimes \cdots \otimes g_{n-1} .
\end{gathered}
$$

We also have

$$
\operatorname{Hom}_{k(G \times G)}\left(k(G \times G) \otimes_{k G} \operatorname{Bar}_{n}(k G) \otimes_{k G} k, k G\right) \simeq \operatorname{Hom}_{k}\left(\overline{k G}^{\otimes n}, k G\right) \simeq \operatorname{Map}\left(\bar{G}^{\times n}, k G\right) .
$$

Using this identification, $H^{*}\left(\operatorname{Hom}_{k(G \times G)}\left(k(G \times G) \otimes_{k G} \operatorname{Bar}_{*}(k G) \otimes_{k G} k, k G\right)\right)$ is given by the following cochain complex:

$$
0 \longrightarrow k G \stackrel{\delta_{0}}{\longrightarrow} \operatorname{Map}(\bar{G}, k G) \stackrel{\delta_{1}}{\longrightarrow} \cdots \longrightarrow \operatorname{Map}\left(\bar{G}^{\times n}, k G\right) \stackrel{\delta_{n}}{\longrightarrow} \cdots,
$$

where the differential is given by

$$
\delta_{0}(x)(g)=g x g^{-1}-x \quad \text { for } x \in k G \text { and } g \in \bar{G},
$$

and for $\varphi: \bar{G}^{\times n} \longrightarrow k G$ and $g_{1}, \cdots, g_{n+1} \in \bar{G}$,

$$
\begin{aligned}
\delta_{n}(\varphi)\left(g_{1}, \cdots, g_{n+1}\right)= & g_{1} \varphi\left(g_{2}, \cdots, g_{n+1}\right) g_{1}^{-1}+ \\
& \sum_{i=1}^{n}(-1)^{i} \varphi\left(g_{1}, \cdots, g_{i} g_{i+1}, \cdots, g_{n+1}\right)+(-1)^{n+1} \varphi\left(g_{1}, \cdots, g_{n}\right) .
\end{aligned}
$$


We will show that the two complexes $k(G \times G) \otimes_{k G} \operatorname{Bar}_{*}(k G) \otimes_{k G} k$ and $\operatorname{Bar}_{*}(k G)$ are isomorphic and therefore there is an isomorphism

(1) $H^{*}\left(\operatorname{Hom}_{k(G \times G)}\left(\operatorname{Bar}_{*}(k G), k G\right)\right) \simeq H^{*}\left(\operatorname{Hom}_{k(G \times G)}\left(k(G \times G) \otimes_{k G} \operatorname{Bar}_{*}(k G) \otimes_{k G} k, k G\right)\right)$.

To do this, we need to construct the comparison maps between the two free resolutions $B a r_{*}(k G)$ and $k(G \times G) \otimes_{k G} \operatorname{Bar}_{*}(k G) \otimes_{k G} k$ of the above $k(G \times G)$-module $k G$. As explained in Section 2 , this is reduced to construct setwise self-homotopys over these resolutions. Our principle here is to choose those setwise self-homotopys so that the computations and results are as simple as possible.

We choose a setwise self-homotopy over $\operatorname{Bar}_{*}(k G)$ as follows:

$$
u_{-1}: k G \rightarrow k G \otimes k G, \quad g \mapsto g \otimes 1,
$$

and for $n \geq 0$,

$$
\begin{gathered}
u_{n}: k G \otimes \overline{k G}^{\otimes n} \otimes k G \longrightarrow k G \otimes \overline{k G}^{\otimes n} \otimes k G, \\
g_{0} \otimes g_{1} \otimes \cdots \otimes g_{n+1} \longmapsto(-1)^{n+1} g_{0} \otimes g_{1} \otimes \cdots \otimes g_{n+1} \otimes 1 .
\end{gathered}
$$

Using $\left\{u_{n}\right\}$ we can construct a comparison map

$$
\alpha_{*}: k(G \times G) \otimes_{k G} \operatorname{Bar}_{*}(k G) \otimes_{k G} k=k G \otimes k G \otimes k \bar{G}^{\otimes *} \longrightarrow \operatorname{Bar}_{*}(k G)=k G \otimes k G^{\otimes *} \otimes k G
$$

as follows (as before we only write down the maps on base elements):

$$
\begin{gathered}
\alpha_{-1}: k G \longrightarrow k G, \quad x \longmapsto x, \\
\alpha_{0}: k G \otimes k G \longrightarrow k G \otimes k G, \quad x \otimes y \longmapsto x \otimes y^{-1}, \\
\alpha_{1}: k G \otimes k G \otimes \overline{k G} \longrightarrow k G \otimes \overline{k G} \otimes k G, \quad x \otimes y \otimes g_{1} \longmapsto-x g_{1} \otimes g_{1}^{-1} \otimes y^{-1}, \\
\cdots \cdots \cdots \\
\alpha_{n}: k G \otimes k G \otimes \overline{k G}^{\otimes n} \longrightarrow k G \otimes \overline{k G}^{\otimes n} \otimes k G, \quad x \otimes y \otimes g_{1} \otimes \cdots \otimes g_{n} \longmapsto \\
(-1)^{\frac{n(n+1)}{2}} x g_{1} \cdots g_{n} \otimes g_{n}^{-1} \otimes \cdots \otimes g_{1}^{-1} \otimes y^{-1} .
\end{gathered}
$$

Similarly, we choose a setwise self-homotopy over $k(G \times G) \otimes_{k G} \operatorname{Bar}_{*}(k G) \otimes_{k G} k$ as follows:

$$
v_{-1}: k G \rightarrow k G \otimes k G, \quad g \mapsto g \otimes 1,
$$

and for $n \geq 0$,

$$
\begin{aligned}
v_{n}: & k G \otimes k G \otimes \overline{k G}^{\otimes n} \longrightarrow k G \otimes k G \otimes \overline{k G}^{\otimes n}, \\
& x \otimes y \otimes g_{1} \otimes \cdots \otimes g_{n} \longmapsto x y^{-1} \otimes 1 \otimes y \otimes g_{1} \otimes \cdots \otimes g_{n} .
\end{aligned}
$$

Using $\left\{v_{n}\right\}$ we can construct a comparison map

$$
\beta_{*}: \operatorname{Bar}_{*}(k G)=k G \otimes k G^{\otimes *} \otimes k G \longrightarrow k(G \times G) \otimes_{k G} \operatorname{Bar}_{*}(k G) \otimes_{k G} k=k G \otimes k G \otimes k G^{\otimes *}
$$

as follows:

$$
\begin{gathered}
\beta_{-1}: k G \longrightarrow k G, \quad x \longmapsto x, \\
\beta_{0}: k G \otimes k G \longrightarrow k G \otimes k G, \quad x \otimes y \longmapsto x \otimes y^{-1}, \\
\beta_{1}: k G \otimes \overline{k G} \otimes k G \longrightarrow k G \otimes k G \otimes \overline{k G}, \quad x \otimes g_{1} \otimes y \longmapsto-x g_{1} \otimes y^{-1} \otimes g_{1}^{-1}, \\
\cdots \cdots \cdots \\
\beta_{n}: k G \otimes \overline{k G}^{\otimes n} \otimes k G \longrightarrow k G \otimes k G \otimes \overline{k G}^{\otimes n}, \quad x \otimes g_{1} \otimes \cdots \otimes g_{n} \otimes y \longmapsto \\
(-1)^{\frac{n(n+1)}{2}} x g_{1} \cdots g_{n} \otimes y^{-1} \otimes g_{n}^{-1} \otimes \cdots \otimes g_{1}^{-1} .
\end{gathered}
$$

It is easy to check that the chain maps $\left\{\alpha_{n}\right\}$ and $\left\{\beta_{n}\right\}$ are inverse to each other, and therefore we get an isomorphism

$$
\begin{aligned}
& \operatorname{Hom}_{k(G \times G)}\left(\operatorname{Bar}_{*}(k G), k G\right) \longrightarrow \operatorname{Hom}_{k(G \times G)}\left(k(G \times G) \otimes_{k G} \operatorname{Bar}_{*}(k G) \otimes_{k G} k, k G\right), \\
& \left(\varphi: \bar{G}^{\times n} \longrightarrow k G\right) \longmapsto\left(\varphi_{1}: \bar{G}^{\times n} \longrightarrow k G\right), \quad \varphi_{1}\left(g_{1}, \cdots, g_{n}\right)=(-1)^{\frac{n(n+1)}{2}} g_{1} \cdots g_{n} \varphi\left(g_{n}^{-1}, \cdots, g_{1}^{-1}\right) .
\end{aligned}
$$

Its inverse is given by

$$
\begin{aligned}
& \operatorname{Hom}_{k(G \times G)}\left(k(G \times G) \otimes_{k G} \operatorname{Bar}_{*}(k G) \otimes_{k G} k, k G\right) \longrightarrow \operatorname{Hom}_{k(G \times G)}\left(\operatorname{Bar}_{*}(k G), k G\right), \\
&\left(\varphi_{1}: \bar{G}^{\times n} \longrightarrow k G\right) \longmapsto\left(\varphi: \bar{G}^{\times n} \longrightarrow k G\right), \quad \varphi\left(g_{1}, \cdots, g_{n}\right)=(-1)^{\frac{n(n+1)}{2}} g_{1} \cdots g_{n} \varphi_{1}\left(g_{n}^{-1}, \cdots, g_{1}^{-1}\right) .
\end{aligned}
$$

Passing to the cohomology, we realize an isomorphism in (1) and its inverse. 
The second step. Since $\left(k(G \times G) k(G \times G) \otimes_{k G}-, H_{o m}(G \times G)\left(k(G \times G) k(G \times G)_{k G},-\right)\right)$ is an adjoint pair, we have an isomorphism (here $k(G \times G)$ is viewed as a right $k G$-module by diagonal action)

$$
\operatorname{Hom}_{k(G \times G)}\left(k(G \times G) \otimes_{k G} \operatorname{Bar}_{*}(k G) \otimes_{k G} k, k G\right) \simeq \operatorname{Hom}_{k G}\left(\operatorname{Bar}_{*}(k G) \otimes_{k G} k,{ }_{c} k G\right) .
$$

Passing to the cohomology, we get an isomorphism

(2) $H^{*}\left(\operatorname{Hom}_{k(G \times G)}\left(k(G \times G) \otimes_{k G} \operatorname{Bar}_{*}(k G) \otimes_{k G} k, k G\right)\right) \simeq H^{*}\left(\operatorname{Hom}_{k G}\left(\operatorname{Bar}_{*}(k G) \otimes_{k G} k,{ }_{c} k G\right)\right)$.

Remind that the right hand side is just the ordinary group cohomology $H^{*}(G, k G)$ of $G$ with coefficients in ${ }_{c} k G$. We also have

$$
\operatorname{Hom}_{k G}\left(\operatorname{Bar}_{n}(k G) \otimes_{k G} k, k G\right) \simeq \operatorname{Hom}_{k G}\left(k G \otimes \overline{k G}^{\otimes n}, k G\right) \simeq \operatorname{Hom}_{k}\left(\overline{k G}^{\otimes n}, k G\right) \simeq \operatorname{Map}\left(\bar{G}^{\times n}, k G\right) .
$$

Using this identification, $H^{*}(G, k G)=H^{*}\left(\operatorname{Hom}_{k G}\left(\operatorname{Bar}_{*}(k G) \otimes_{k G} k, k G\right)\right)$ is given by the following cochain complex:

$$
0 \longrightarrow k G \stackrel{\delta_{0}}{\longrightarrow} \operatorname{Map}(\bar{G}, k G) \stackrel{\delta_{1}}{\longrightarrow} \cdots \longrightarrow \operatorname{Map}\left(\bar{G}^{\times n}, k G\right) \stackrel{\delta_{n}}{\longrightarrow} \cdots,
$$

where the differential is given by

$$
\delta_{0}(x)(g)=g x g^{-1}-x \quad(\text { for } x \in k G \text { and } g \in \bar{G}),
$$

and (for $\varphi: \bar{G}^{\times n} \longrightarrow k G$ and $g_{1}, \cdots, g_{n+1} \in \bar{G}$ )

$$
\begin{aligned}
\delta_{n}(\varphi)\left(g_{1}, \cdots, g_{n+1}\right)= & g_{1} \varphi\left(g_{2}, \cdots, g_{n+1}\right) g_{1}^{-1}+ \\
& \sum_{i=1}^{n}(-1)^{i} \varphi\left(g_{1}, \cdots, g_{i} g_{i+1}, \cdots, g_{n+1}\right)+(-1)^{n+1} \varphi\left(g_{1}, \cdots, g_{n}\right) .
\end{aligned}
$$

So formally the left hand side and the right hand side in (2) are identical, though they have different meaning. It is also easy to check that under the above identifications, the adjoint isomorphisms are identity maps:

$$
\begin{gathered}
\operatorname{Hom}_{k(G \times G)}\left(k(G \times G) \otimes_{k G} \operatorname{Bar}_{*}(k G) \otimes_{k G} k, k G\right) \longrightarrow \operatorname{Hom}_{k G}\left(\operatorname{Bar}_{*}(k G) \otimes_{k G} k,{ }_{c} k G\right), \\
\left(\varphi_{1}: \bar{G}^{\times n} \longrightarrow k G\right) \longmapsto\left(\varphi_{2}: \bar{G}^{\times n} \longrightarrow k G\right), \quad \varphi_{2}\left(g_{1}, \cdots, g_{n}\right)=\varphi_{1}\left(g_{1}, \cdots, g_{n}\right) .
\end{gathered}
$$

Its inverse is given by

$$
\begin{gathered}
\operatorname{Hom}_{k G}\left(\operatorname{Bar}_{*}(k G) \otimes_{k G} k,{ }_{c} k G\right) \longrightarrow \operatorname{Hom}_{k(G \times G)}\left(k(G \times G) \otimes_{k G} \operatorname{Bar}_{*}(k G) \otimes_{k G} k, k G\right), \\
\left(\varphi_{2}: \bar{G}^{\times n} \longrightarrow k G\right) \longmapsto\left(\varphi_{1}: \bar{G}^{\times n} \longrightarrow k G\right), \quad \varphi_{1}\left(g_{1}, \cdots, g_{n}\right)=\varphi_{2}\left(g_{1}, \cdots, g_{n}\right) .
\end{gathered}
$$

Passing to the cohomology, we realize an isomorphism in (2) and its inverse.

The third step. We choose a complete set $X$ of representatives of the conjugacy classes in the finite group $G$. Take $x \in X$. Then $C_{x}=\left\{g x g^{-1} \mid g \in G\right\}$ is the conjugacy class corresponding to $x$ and $C_{G}(x)=\left\{g \in G \mid g x g^{-1}=x\right\}$ is the centralizer subgroup. Clearly the $k$-space $k C_{x}$ generated by the elements in $C_{x}$ is a left $k G$-module under the conjugation action. We choose a right coset decomposition of $C_{G}(x)$ in $G: G=C_{G}(x) \gamma_{1, x} \cup C_{G}(x) \gamma_{2, x} \cup \cdots \cup C_{G}(x) \gamma_{n_{x}, x}$ (equivalently, $G=\gamma_{1, x}^{-1} C_{G}(x) \cup \gamma_{2, x}^{-1} C_{G}(x) \cup \cdots \cup \gamma_{n_{x}, x}^{-1} C_{G}(x)$ is a left coset decomposition of $C_{G}(x)$ in $\left.G\right)$, and such that $C_{x}=\left\{x=\gamma_{1, x}^{-1} x \gamma_{1, x}, \gamma_{2, x}^{-1} x \gamma_{2, x}, \cdots, \gamma_{n_{x}, x}^{-1} x \gamma_{n_{x}, x}\right\}$. (We will always take $\gamma_{1, x}=1$, and we write $x_{i}$ for $\gamma_{i, x}^{-1} x \gamma_{i, x}$.) Then we have the following $k G$-module isomorphisms:

$$
{ }_{c} k C_{x} \simeq \operatorname{Ind}_{C_{G}(x)}^{G} k={ }_{k G} k G \otimes_{k C_{G}(x)} k, \quad x_{i} \longmapsto \gamma_{i, x}^{-1} \otimes 1,
$$

$$
{ }_{c} k C_{x} \simeq \operatorname{Coind}_{C_{G}(x)}^{G} k=\operatorname{Hom}_{k C_{G}(x)}\left({ }_{k C_{G}(x)} k G_{k G},{ }_{k C_{G}(x)} k\right), \quad x_{i} \longmapsto \gamma_{i}: k G \longrightarrow k, \gamma_{i}\left(\gamma_{j, x}\right)=\delta_{i j},
$$

where in the first isomorphism, the left $k G$-module structure on $k G$ is the usual left multiplication and the right $k C_{G}(x)$-module structure on $k G$ is given by restriction, and $k$ is the trivial $k C_{G}(x)$-module, and the same as in the second isomorphism.

In the second step, we have arrived at the ordinary group cohomology $H^{*}(G, k G)$ of $G$ with coefficients in ${ }_{c} k G$. This ${ }_{c} k G$ has a $k G$-module decomposition:

$$
{ }_{c} k G=\bigoplus_{x \in X}{ }_{c} k C_{x} .
$$


Denote by $\pi_{x}: k G \longrightarrow k C_{x}$ and $i_{x}: k C_{x} \longrightarrow k G$ the canonical projection and the canonical injection, respectively. Then we have the following isomorphism

$$
\begin{gathered}
\operatorname{Hom}_{k G}\left(\operatorname{Bar}_{*}(k G) \otimes_{k G} k,{ }_{c} k G\right) \longrightarrow \bigoplus_{x \in X} \operatorname{Hom}_{k G}\left(\operatorname{Bar}_{*}(k G) \otimes_{k G} k,{ }_{c} k C_{x}\right), \\
\left(\varphi_{2}: \bar{G}^{\times n} \longrightarrow k G\right) \longmapsto \varphi_{3}=\left\{\varphi_{3, x} \mid x \in X\right\}, \text { where } \varphi_{3, x}=\pi_{x} \varphi_{2}: \bar{G}^{\times n} \longrightarrow k C_{x} .
\end{gathered}
$$

Its inverse is given by

$$
\begin{aligned}
& \bigoplus_{x \in X} \operatorname{Hom}_{k G}\left(\operatorname{Bar}_{*}(k G) \otimes_{k G} k, k C_{x}\right) \longrightarrow \operatorname{Hom}_{k G}\left(\operatorname{Bar}_{*}(k G) \otimes_{k G} k, k G\right), \\
& \varphi_{3}=\left\{\varphi_{3, x}: \bar{G}^{\times n} \longrightarrow k C_{x} \mid x \in X\right\} \longmapsto\left(\varphi_{2}=\sum_{x \in X} i_{x} \varphi_{3, x}: \bar{G}^{\times n} \longrightarrow k G\right) .
\end{aligned}
$$

Passing to the cohomology, we realize an isomorphism:

$$
\text { (3) } H^{*}\left(G,{ }_{c} k G\right) \simeq \bigoplus_{x \in X} H^{*}\left(G,{ }_{c} k C_{x}\right) .
$$

The fourth step. We have stated in the third step the following $k G$-module isomorphism

$$
{ }_{c} k C_{x} \simeq \operatorname{Hom}_{k C_{G}(x)}(k G, k), \quad x_{i} \longmapsto \gamma_{i}: k G \longrightarrow k, \gamma_{i}\left(\gamma_{j, x}\right)=\delta_{i j}
$$

Therefore we have the following isomorphism

$$
\begin{gathered}
\operatorname{Hom}_{k G}\left(\operatorname{Bar}_{*}(k G) \otimes_{k G} k,{ }_{c} k C_{x}\right) \longrightarrow \operatorname{Hom}_{k G}\left(\operatorname{Bar}_{*}(k G) \otimes_{k G} k, \operatorname{Hom}_{k C_{G}(x)}(k G, k)\right), \\
\left(\varphi_{3, x}: \bar{G}^{\times n} \longrightarrow k C_{x}\right) \longmapsto\left(\varphi_{4, x}: \bar{G}^{\times n} \longrightarrow \operatorname{Hom}_{k C_{G}(x)}(k G, k)\right),
\end{gathered}
$$

where if we write $\varphi_{3, x}\left(g_{1}, g_{2}, \cdots, g_{n}\right)=\sum_{i=1}^{n_{x}} a_{i, x} x_{i}$, then $\varphi_{4, x}\left(g_{1}, g_{2}, \cdots, g_{n}\right)$ maps $\gamma_{i, x}$ to $a_{i, x}$ for any $i$. The inverse isomorphism is given by

$$
\begin{gathered}
\operatorname{Hom}_{k G}\left(\operatorname{Bar}_{*}(k G) \otimes_{k G} k, \operatorname{Hom}_{k C_{G}(x)}(k G, k)\right) \longrightarrow \operatorname{Hom}_{k G}\left(\operatorname{Bar}_{*}(k G) \otimes_{k G} k,{ }_{c} k C_{x}\right), \\
\left(\varphi_{4, x}: \bar{G}^{\times n} \longrightarrow \operatorname{Hom}_{k C_{G}(x)}(k G, k)\right) \longmapsto\left(\varphi_{3, x}: \bar{G}^{\times n} \longrightarrow k C_{x}\right),
\end{gathered}
$$

where if $\varphi_{4, x}\left(g_{1}, g_{2}, \cdots, g_{n}\right)$ maps $\gamma_{i, x}$ to $a_{i, x}$ for any $i$, then $\varphi_{3, x}\left(g_{1}, g_{2}, \cdots, g_{n}\right)=\sum_{i=1}^{n_{x}} a_{i, x} x_{i}$. Passing to the cohomology, we realize an isomorphism:

$$
\text { (4) } H^{*}\left(G, k C_{x}\right) \simeq H^{*}\left(\operatorname{Hom}_{k G}\left(\operatorname{Bar}_{*}(k G) \otimes_{k G} k, H o m_{k C_{G}(x)}(k G, k)\right)\right) \text {. }
$$

The fifth step. Since $\left(k G \otimes_{k G}-\operatorname{Hom}_{k C_{G}(x)}(k G,-)\right)$ is an adjoint pair (restriction and coinduction), we have the following isomorphism

$$
\operatorname{Hom}_{k G}\left(\operatorname{Bar}_{*}(k G) \otimes_{k G} k, \operatorname{Hom}_{k C_{G}(x)}(k G, k)\right) \longrightarrow \operatorname{Hom}_{k C_{G}(x)}\left(\operatorname{Bar}_{*}(k G) \otimes_{k G} k, k\right) .
$$

Passing to the cohomology, we get an isomorphism

(5) $H^{*}\left(\operatorname{Hom}_{k G}\left(\operatorname{Bar}_{*}(k G) \otimes_{k G} k, \operatorname{Hom}_{k C_{G}(x)}(k G, k)\right)\right) \simeq H^{*}\left(\operatorname{Hom}_{k C_{G}(x)}\left(\operatorname{Bar}_{*}(k G) \otimes_{k G} k, k\right)\right)$,

where the right hand side is isomorphic to the ordinary group cohomology $H^{*}\left(C_{G}(x), k\right)$ of $C_{G}(x)$ with coefficients in the trivial module $k$. Since there are $k C_{G}(x)$-module isomorphisms

$$
\operatorname{Bar}_{*}(k G) \otimes_{k G} k \simeq \bigoplus_{i=1}^{n_{x}} k C_{G}(x) \gamma_{i, x} \otimes \overline{k G}^{\otimes *}
$$

we have

$$
\operatorname{Hom}_{k C_{G}(x)}\left(\operatorname{Bar}_{*}(k G) \otimes_{k G} k, k\right) \simeq \operatorname{Hom}_{k}\left(\bigoplus_{i=1}^{n_{x}} k \gamma_{i, x} \otimes \overline{k G}^{\otimes n}, k\right) \simeq \operatorname{Map}\left(S_{x} \times \bar{G}^{\times n}, k\right),
$$

where $S_{x}=\left\{\gamma_{1, x}, \cdots, \gamma_{n_{x}, x}\right\}$ (cf. The third step). Using this identification, the adjoint isomorphism is given by

$$
\begin{gathered}
\operatorname{Hom}_{k G}\left(\operatorname{Bar}_{*}(k G) \otimes_{k G} k, \operatorname{Hom}_{k C_{G}(x)}(k G, k)\right) \longrightarrow \operatorname{Hom}_{k C_{G}(x)}\left(\operatorname{Bar}_{*}(k G) \otimes_{k G} k, k\right), \\
\left(\varphi_{4, x}: \bar{G}^{\times n} \longrightarrow \operatorname{Hom}_{k C_{G}(x)}(k G, k)\right) \longmapsto\left(\varphi_{5, x}: S_{x} \times \bar{G}^{\times n} \longrightarrow k\right),
\end{gathered}
$$


where if $\varphi_{4, x}\left(g_{1}, g_{2}, \cdots, g_{n}\right)$ maps $\gamma_{i, x}$ to $a_{i, x}$ for any $i$, then $\varphi_{5, x}\left(\gamma_{i, x}, g_{1}, g_{2}, \cdots, g_{n}\right)=a_{i, x}$ for any $i$. The inverse isomorphism is given by

$$
\begin{gathered}
\operatorname{Hom}_{k C_{G}(x)}\left(\operatorname{Bar}_{*}(k G) \otimes_{k G} k, k\right) \longrightarrow \operatorname{Hom}_{k G}\left(\operatorname{Bar}_{*}(k G) \otimes_{k G} k, \operatorname{Hom}_{k C_{G}(x)}(k G, k)\right), \\
\left(\varphi_{5, x}: S_{x} \times \bar{G}^{\times n} \longrightarrow k\right) \longmapsto\left(\varphi_{4, x}: \bar{G}^{\times n} \longrightarrow \operatorname{Hom}_{k C_{G}(x)}(k G, k)\right),
\end{gathered}
$$

where if $\varphi_{5, x}\left(\gamma_{i, x}, g_{1}, g_{2}, \cdots, g_{n}\right)=a_{i, x}$ for any $i$, then $\varphi_{4, x}\left(g_{1}, g_{2}, \cdots, g_{n}\right)$ maps $\gamma_{i, x}$ to $a_{i, x}$ for any $i$. Passing to the cohomology, we realize an isomorphism in (5) and its inverse.

The sixth step. In the fifth step, we have arrived at the ordinary group cohomology $H^{*}\left(C_{G}(x), k\right)$ of $C_{G}(x)$ with coefficients in the trivial module $k$, where $H^{*}\left(C_{G}(x), k\right)$ is computed by the cochain complex $\operatorname{Hom}_{k C_{G}(x)}\left(\operatorname{Bar}_{*}(k G) \otimes_{k G} k, k\right)$. By the identification in fifth step, this is given by the following cochain complex:

$$
0 \longrightarrow k^{\times n_{x}} \stackrel{\delta_{0}}{\longrightarrow} \operatorname{Map}\left(S_{x} \times \bar{G}, k\right) \stackrel{\delta_{1}}{\longrightarrow} \cdots \longrightarrow \operatorname{Map}\left(S_{x} \times \bar{G}^{\times n}, k\right) \stackrel{\delta_{n}}{\longrightarrow} \cdots,
$$

where the differential is given by $\delta_{0}\left(\left\{a_{i, x}\right\}\right)\left(\left(\gamma_{j, x}, g_{1}\right)\right)=a_{s_{j}, x}-a_{j, x}$, such that $a_{s_{j}, x}$ is determined as follows: for $\left\{a_{i, x}\right\} \in k^{\times n_{x}}, \gamma_{j, x} \in S_{x}, g_{1} \in \bar{G}$, we have

$$
\gamma_{j, x} g_{1}=h_{j, 1} \gamma_{s_{j}, x} \text { for some } h_{j, 1} \in C_{G}(x) \text { and for some } 1 \leq s_{j} \leq n_{x},
$$

and (for $\varphi: S_{x} \times \bar{G}^{\times n} \longrightarrow k, \gamma_{j, x} \in S_{x}, g_{1}, \cdots, g_{n+1} \in \bar{G}$ such that $\gamma_{j, x} g_{1}=h_{j, 1} \gamma_{s_{j}, x}$ )

$$
\begin{aligned}
& \delta_{n}(\varphi)\left(\gamma_{j, x}, g_{1}, \cdots, g_{n+1}\right)=\varphi\left(\gamma_{s_{j}, x}, g_{2}, \cdots, g_{n+1}\right)+ \\
& \sum_{i=1}^{n}(-1)^{i} \varphi\left(\gamma_{j, x}, g_{1}, \cdots, g_{i} g_{i+1}, \cdots, g_{n+1}\right)+(-1)^{n+1} \varphi\left(\gamma_{j, x}, g_{1}, \cdots, g_{n}\right) .
\end{aligned}
$$

(Remark that for a fixed $g_{1} \in \bar{G},\left\{s_{1}, s_{2}, \cdots, s_{n_{x}}\right\}$ is a permutation of $\left\{1,2, \cdots, n_{x}\right\}$.)

The above computation for $H^{*}\left(C_{G}(x), k\right)$ uses the projective resolution $\operatorname{Bar}_{*}(k G) \otimes_{k G} k$ of the trivial $k C_{G}(x)$-module $k$, which is identified as the following complex (It is in fact a projective resolution of the trivial $k G$-module $k$, but we view it as a complex of $k C_{G}(x)$-modules by restriction)

$$
\cdots \longrightarrow k G \otimes \overline{k G}^{\otimes n} \stackrel{d_{n}}{\longrightarrow} \cdots \longrightarrow k G \otimes \overline{k G} \stackrel{d_{1}}{\longrightarrow} k G \stackrel{d_{0}}{\longrightarrow} k \longrightarrow 0,
$$

where the differential is given by

and (for $g_{0} \in G, g_{1}, \cdots, g_{n} \in \bar{G}$ )

$$
d_{0}\left(g_{0}\right)=1 \quad\left(\text { for } g_{0} \in G\right)
$$

$$
\begin{aligned}
& d_{n}\left(g_{0}, g_{1}, \cdots, g_{n}\right)=g_{0} g_{1} \otimes g_{2} \otimes \cdots \otimes g_{n}+ \\
& \qquad \sum_{i=1}^{n-1}(-1)^{i} g_{0} \otimes \cdots \otimes g_{i} g_{i+1} \otimes \cdots \otimes g_{n}+(-1)^{n} g_{0} \otimes g_{1} \otimes \cdots \otimes g_{n-1} .
\end{aligned}
$$

We now use another projective resolution $\operatorname{Bar}_{*}\left(k C_{G}(x)\right) \otimes_{k C_{G}(x)} k$ of the trivial $k C_{G}(x)$-module $k$, which is identified as the following complex

$$
\cdots \longrightarrow k C_{G}(x) \otimes{\overline{k C_{G}(x)}}^{\otimes n} \stackrel{d_{n}}{\longrightarrow} \cdots \longrightarrow k C_{G}(x) \otimes \overline{k C_{G}(x)} \stackrel{d_{1}}{\longrightarrow} k C_{G}(x) \stackrel{d_{0}}{\longrightarrow} k \longrightarrow 0,
$$

where the differential is given by

$$
d_{0}\left(h_{0}\right)=1 \quad\left(\text { for } h_{0} \in C_{G}(x)\right)
$$

and (for $h_{0} \in C_{G}(x), h_{1}, \cdots, h_{n} \in \overline{C_{G}(x)}$ )

$$
\begin{aligned}
& d_{n}\left(h_{0}, h_{1}, \cdots, h_{n}\right)=h_{0} h_{1} \otimes h_{2} \otimes \cdots \otimes h_{n}+ \\
& \qquad \sum_{i=1}^{n-1}(-1)^{i} h_{0} \otimes \cdots \otimes h_{i} h_{i+1} \otimes \cdots \otimes h_{n}+(-1)^{n} h_{0} \otimes h_{1} \otimes \cdots \otimes h_{n-1} .
\end{aligned}
$$

We have

$$
\operatorname{Hom}_{k C_{G}(x)}\left(\operatorname{Bar}_{*}\left(k C_{G}(x)\right) \otimes_{k C_{G}(x)} k, k\right) \simeq \operatorname{Map}\left({\overline{C_{G}(x)}}^{\times n}, k\right),
$$

so $H^{*}\left(C_{G}(x), k\right)$ can also be computed by the following cochain complex

$$
0 \longrightarrow k \stackrel{\delta_{0}}{\longrightarrow} \operatorname{Map}\left(\overline{C_{G}(x)}, k\right) \stackrel{\delta_{1}}{\longrightarrow} \cdots \longrightarrow \operatorname{Map}\left({\overline{C_{G}(x)}}^{\times n}, k\right) \stackrel{\delta_{n}}{\longrightarrow} \cdots,
$$


where the differential is given by

$$
\delta_{0}(a)\left(h_{1}\right)=0 \quad\left(\text { for } a \in k, h_{1} \in \overline{C_{G}(x)}\right)
$$

and (for $\varphi:{\overline{C_{G}(x)}}^{\times n} \longrightarrow k, h_{1}, \cdots, h_{n+1} \in \overline{C_{G}(x)}$ )

$$
\begin{aligned}
& \delta_{n}(\varphi)\left(h_{1}, \cdots, h_{n+1}\right)=\varphi\left(h_{2}, \cdots, h_{n+1}\right)+ \\
& \qquad \sum_{i=1}^{n}(-1)^{i} \varphi\left(h_{1}, \cdots, h_{i} h_{i+1}, \cdots, h_{n+1}\right)+(-1)^{n+1} \varphi\left(h_{1}, \cdots, h_{n}\right) .
\end{aligned}
$$

Clearly, we have

(6) $H^{*}\left(\operatorname{Hom}_{k C_{G}(x)}\left(\operatorname{Bar}_{*}(k G) \otimes_{k G} k, k\right)\right) \simeq H^{*}\left(\operatorname{Hom}_{k C_{G}(x)}\left(\operatorname{Bar}_{*}\left(k C_{G}(x)\right) \otimes_{k C_{G}(x)} k, k\right)\right)$.

To give an explicit isomorphism in (6), we need to construct the comparison maps between two projective resolutions $B a r_{*}(k G) \otimes_{k G} k$ and $B a r_{*}\left(k C_{G}(x)\right) \otimes_{k C_{G}(x)} k$ of the trivial $k C_{G}(x)$-module $k$.

The comparison map from $\operatorname{Bar}_{*}\left(k C_{G}(x)\right) \otimes_{k C_{G}(x)} k$ to $\operatorname{Bar}_{*}(k G) \otimes_{k G} k$ is just the inclusion map

$$
\iota: k C_{G}(x) \otimes{\overline{k C_{G}(x)}}^{\otimes n} \hookrightarrow k G \otimes \overline{k G}^{\otimes n} .
$$

This is obvious or can be obtained using a setwise self-homotopy on $\operatorname{Bar}_{*}(k G) \otimes_{k G} k$ (see below for its explicit form).

To construct the comparison map on the reverse direction, we use a setwise self-homotopy over $k C_{G}(x) \otimes{\overline{k C_{G}(x)}}^{\otimes *}$ as follows (for $\left.h_{0} \in C_{G}(x), h_{1}, \cdots, h_{n} \in \overline{C_{G}(x)}\right)$ :

$$
\begin{gathered}
k C_{G}(x) \otimes{\overline{k C_{G}(x)}}^{\otimes n} \longrightarrow k C_{G}(x) \otimes{\overline{k C_{G}(x)}}^{\otimes n+1}, \\
h_{0} \otimes h_{1} \otimes \cdots \otimes h_{n} \longmapsto 1 \otimes h_{0} \otimes h_{1} \otimes \cdots \otimes h_{n} .
\end{gathered}
$$

Then we get a comparison map

$$
\rho: \operatorname{Bar}_{*}(k G) \otimes_{k G} k \longrightarrow \operatorname{Bar}_{*}\left(k C_{G}(x)\right) \otimes_{k C_{G}(x)} k
$$

as follows:

$$
\begin{gathered}
\rho_{-1}: k \longrightarrow k, \quad 1 \longmapsto 1, \\
\rho_{0}: k G \longrightarrow k C_{G}(x), \quad h \gamma_{i, x} \longmapsto h, \text { for } h \in C_{G}(x), \\
\rho_{1}: k G \otimes \overline{k G} \longrightarrow k C_{G}(x) \otimes \overline{k C_{G}(x)}, \quad h \gamma_{i, x} \otimes g_{1} \longmapsto h \otimes h_{i, 1}, \\
\text { where } \gamma_{i, x} g_{1}=h_{i, 1} \gamma_{s_{i}, x} \text { for } h_{i, 1} \in \overline{C_{G}(x)},
\end{gathered}
$$

$$
\text { .......... }
$$

$$
\rho_{n}: k G \otimes \overline{k G}^{\otimes n} \longrightarrow k C_{G}(x) \otimes{\overline{k C_{G}(x)}}^{\otimes n}, \quad h \gamma_{i, x} \otimes g_{1} \otimes \cdots \otimes g_{n} \longmapsto h \otimes h_{i, 1} \otimes \cdots \otimes h_{i, n},
$$

where $h_{i, 1}, \cdots, h_{i, n} \in \overline{C_{G}(x)}$ are determined by the sequence $\left\{g_{1}, \cdots, g_{n}\right\}$ as follows:

$$
\gamma_{i, x} g_{1}=h_{i, 1} \gamma_{s_{i}^{1}, x}, \quad \gamma_{s_{i}^{1}, x} g_{2}=h_{i, 2} \gamma_{s_{i}^{2}, x}, \quad \cdots, \quad \gamma_{s_{i}^{n-1}, x} g_{n}=h_{i, n} \gamma_{s_{i}^{n}, x} .
$$

Notice that $\rho \circ \iota=I d$ and $\iota \circ \rho \neq I d$. It follows that we have two homomorphisms:

$$
\begin{gathered}
\operatorname{Hom}_{k C_{G}(x)}\left(\operatorname{Bar}_{*}(k G) \otimes_{k G} k, k\right) \longrightarrow \operatorname{Hom}_{k C_{G}(x)}\left(\operatorname{Bar}_{*}\left(k C_{G}(x)\right) \otimes_{k C_{G}(x)} k, k\right), \\
\left(\varphi_{5, x}: S_{x} \times \bar{G}^{\times n} \longrightarrow k\right) \longmapsto\left(\varphi_{6, x}: \bar{C}_{G}(x)\right. \\
\text { where } a_{1, x} \text { is the cofficients of } x \text { in } \varphi_{3, x}\left(h_{1}, \cdots, h_{n}\right)=\sum_{i=1}^{n_{x}} a_{i, x} x_{i} ;
\end{gathered}
$$

and

$$
\begin{aligned}
& \quad \operatorname{Hom}_{k C_{G}(x)}\left(\operatorname{Bar}_{*}\left(k C_{G}(x)\right) \otimes_{k C_{G}(x)} k, k\right) \longrightarrow \operatorname{Hom}_{k C_{G}(x)}\left(\operatorname{Bar}_{*}(k G) \otimes_{k G} k, k\right), \\
& \left(\varphi_{6, x}: \overline{C_{G}(x)} \times n \longrightarrow k\right) \longmapsto\left(\varphi_{5, x}: S_{x} \times \bar{G}^{\times n} \longrightarrow k\right), \quad \varphi_{5, x}\left(\gamma_{i, x}, g_{1}, \cdots, g_{n}\right)=\varphi_{6, x}\left(h_{i, 1}, \cdots, h_{i, n}\right), \\
& \text { where for } h_{i, 1}, \cdots, h_{i, n} \in \overline{C_{G}(x)} \text { are determined by the sequence }\left\{g_{1}, \cdots, g_{n}\right\} \text { as follows: }
\end{aligned}
$$

$$
\gamma_{i, x} g_{1}=h_{i, 1} \gamma_{s_{i}^{1}, x}, \quad \gamma_{s_{i}^{1}, x} g_{2}=h_{i, 2} \gamma_{s_{i}^{2}, x}, \quad \cdots, \quad \gamma_{s_{i}^{n-1}, x} g_{n}=h_{i, n} \gamma_{s_{i}^{n}, x} .
$$

Since both $\iota$ and $\rho$ induce the identity map $1: k \longrightarrow k$, by Lemma 2.3. we have inverse isomorphisms between $H^{*}\left(\operatorname{Hom}_{k C_{G}(x)}\left(\operatorname{Bar}_{*}(k G) \otimes_{k G} k, k\right)\right)$ and $H^{*}\left(\operatorname{Hom}_{k C_{G}(x)}\left(\operatorname{Bar}_{*}\left(k C_{G}(x)\right) \otimes_{k C_{G}(x)} k, k\right)\right)$. The correspondence is induced by $\varphi_{5, x} \longleftrightarrow \varphi_{6, x}$, as we stated above. So we realize an isomorphism in (6) and its inverse. 
Summarizing the above six steps, we get the following main result in this section.

Theorem 5.1. Let $k$ be a field and $G$ a finite group. Consider the additive decomposition of Hochschild cohomology ring of the group algebra $k G$ :

$$
H H^{*}(k G) \simeq \bigoplus_{x \in X} H^{*}\left(C_{G}(x), k\right),
$$

where $X$ is a set of representatives of conjugacy classes of elements of $G$ and $C_{G}(x)$ is the centralizer subgroup of $G$. We compute the Hochschild cohomology $H H^{*}(k G)=H^{*}\left(H_{o m}(G \times G)\left(\operatorname{Bar}_{*}(k G), k G\right)\right)$ by the classical normalized bar resolution, and we compute the group cohomology $H^{*}\left(C_{G}(x), k\right)$ by $H^{*}\left(\operatorname{Hom}_{k C_{G}(x)}\left(\operatorname{Bar}_{*}\left(k C_{G}(x)\right) \otimes_{k C_{G}(x)} k, k\right)\right)$. Then, we can realize an isomorphism in additive decomposition as follows:

$$
\begin{aligned}
& H H^{*}(k G) \stackrel{\sim}{\longrightarrow} \bigoplus_{x \in X} H^{*}\left(C_{G}(x), k\right), \\
& {\left[\varphi: \bar{G}^{\times n} \longrightarrow k G\right] \longmapsto[\widehat{\varphi}]=\bigoplus_{x \in X}\left[\widehat{\varphi}_{x}\right], \quad \widehat{\varphi}_{x}:{\overline{C_{G}(x)}}^{\times n} \longrightarrow k} \\
& \widehat{\varphi}_{x}\left(h_{1}, \cdots, h_{n}\right)=a_{1, x}, \text { where } \pi_{x}\left((-1)^{\frac{n(n+1)}{2}} h_{1} \cdots h_{n} \varphi\left(h_{n}^{-1}, \cdots, h_{1}^{-1}\right)\right)=\sum_{i=1}^{n_{x}} a_{i, x} x_{i} \text {. }
\end{aligned}
$$

In other word, $\widehat{\varphi}_{x}\left(h_{1}, \cdots, h_{n}\right)$ is just the coefficient of $x$ in $(-1)^{\frac{n(n+1)}{2}} h_{1} \cdots h_{n} \varphi\left(h_{n}^{-1}, \cdots, h_{1}^{-1}\right) \in k G$. The inverse of the above isomorphism is given as follows:

$$
\begin{aligned}
& \bigoplus_{x \in X} H^{*}\left(C_{G}(x), k\right) \stackrel{\sim}{\longrightarrow} H H^{*}(k G), \\
& {[\widehat{\varphi}]=\bigoplus_{x \in X}\left[\widehat{\varphi}_{x}\right], \quad \widehat{\varphi}_{x}:{\overline{C_{G}(x)}}^{\times n} \longrightarrow k \longmapsto\left[\varphi: \bar{G}^{\times n} \longrightarrow k G\right],} \\
& \varphi\left(g_{1}, \cdots, g_{n}\right)=(-1)^{\frac{n(n+1)}{2}} g_{1} \cdots g_{n} \sum_{x \in X} \sum_{i=1}^{n_{x}} \widehat{\varphi}_{x}\left(h_{i, 1}^{\prime}, \cdots, h_{i, n}^{\prime}\right) x_{i},
\end{aligned}
$$

where for $x \in X, h_{i, 1}^{\prime}, \cdots, h_{i, n}^{\prime} \in \overline{C_{G}(x)}$ are determined by the sequence $\left\{g_{n}^{-1}, \cdots, g_{1}^{-1}\right\}$ as follows:

$$
\gamma_{i, x} g_{n}^{-1}=h_{i, 1}^{\prime} \gamma_{s_{i}^{1}, x}, \quad \gamma_{s_{i}^{1}, x} g_{n-1}^{-1}=h_{i, 2}^{\prime} \gamma_{s_{i}^{2}, x}, \quad \cdots, \quad \gamma_{s_{i}^{n-1}, x} g_{1}^{-1}=h_{i, n}^{\prime} \gamma_{s_{i}^{n}, x}
$$

Proof This is a direct consequence by applying the above isomorphisms from (1) to (6) and their inverses. For an element $\varphi: \bar{G}^{\times n} \longrightarrow k G$ in the $n$-th term $C^{n}(k G) \simeq \operatorname{Map}\left(\bar{G}^{\times n}, k G\right)$ of the Hochschild cohomology complex, $[\varphi]$ denotes the corresponding element in the Hochschild cohomology group $H H^{n}(k G)$. Note that the elements $h_{i, 1}^{\prime}, \cdots, h_{i, n}^{\prime}$ depend on $x \in X$ and the sequence $\left\{g_{n}^{-1}, \cdots, g_{1}^{-1}\right\}$. For the simplicity of notations, we avoid to write them down explicitly.

Remark 5.2. (a) The correspondence in Theorem 5.1 makes use of the same line employed by Siegel and Witherspoon in [16]. The difference is: they realize each step between cohomology groups using standard operations like restriction, induction, conjugation, etc., while we construct maps directly in each step on the cohomology complex level.

(b) In [16, as the authors proved that $H H^{*}(k G) \simeq H^{*}\left(G,{ }_{C} k G\right)$ as graded algebras, they concentrated on $H^{*}\left(G,{ }_{c} k G\right)$ instead of $H H^{*}(k G)$ in most part of their paper. If we only consider the isomorphisms (2)-(5), then the correspondence in Theorem 5.1 become simpler:

$$
\begin{gathered}
H^{*}\left(G,{ }_{c} k G\right) \stackrel{\sim}{\longrightarrow} \bigoplus_{x \in X} H^{*}\left(C_{G}(x), k\right), \\
{\left[\varphi: \bar{G}^{\times n} \longrightarrow k G\right] \longmapsto[\widehat{\varphi}]=\bigoplus_{x \in X}\left[\widehat{\varphi}_{x}\right], \quad \widehat{\varphi}_{x}:{\overline{C_{G}(x)}}^{\times n} \longrightarrow k,} \\
\widehat{\varphi}_{x}\left(h_{1}, \cdots, h_{n}\right)=a_{1, x}, \text { the coefficient of } x \text { in } \varphi\left(h_{1}, \cdots, h_{n}\right) \in k G ; \\
\bigoplus_{x \in X} H^{*}\left(C_{G}(x), k\right) \stackrel{\sim}{\longrightarrow} H^{*}\left(G,{ }_{c} k G\right),
\end{gathered}
$$




$$
\begin{gathered}
{[\widehat{\varphi}]=\bigoplus_{x \in X}\left[\widehat{\varphi}_{x}\right], \quad \widehat{\varphi}_{x}:{\overline{C_{G}(x)}}^{\times n} \longrightarrow k \longmapsto\left[\varphi: \bar{G}^{\times n} \longrightarrow k G\right],} \\
\varphi\left(g_{1}, \cdots, g_{n}\right)=\sum_{x \in X} \sum_{i=1}^{n_{x}} \widehat{\varphi}_{x}\left(h_{i, 1}, \cdots, h_{i, n}\right) x_{i},
\end{gathered}
$$

where for $x \in X, h_{i, 1}, \cdots, h_{i, n} \in \overline{C_{G}(x)}$ are determined by the sequence $\left\{g_{1}, \cdots, g_{n}\right\}$ as follows:

$$
\gamma_{i, x} g_{1}=h_{i, 1} \gamma_{s_{i}^{1}, x}, \quad \gamma_{s_{i}^{1}, x} g_{2}=h_{i, 2} \gamma_{s_{i}^{2}, x}, \quad \cdots, \quad \gamma_{s_{i}^{n-1}, x} g_{n}=h_{i, n} \gamma_{s_{i}^{n}, x} .
$$

\section{Another ReAlization of the ADditive DeComposition}

In 4, Cibils and Solotar constructed a subcomplex of the Hochschild cohomology complex for each conjugacy class, and then they showed that for a finite abelian group, the subcomplex is isomorphic to the complex computing group cohomology. We will generalize this to any finite group: for each conjugacy class, this complex computes the cohomology of the corresponding centralizer subgroup. As a result, we give a second way to realize the additive decomposition.

As before, let $k$ be a field and $G$ a finite group. Recall that the Hochschild cohomology $H H^{*}(k G)$ of the group algebra $k G$ can be computed by the following (cochain) complex:

$$
0 \longrightarrow k G \stackrel{\delta_{0}}{\longrightarrow} \operatorname{Map}(\bar{G}, k G) \stackrel{\delta_{1}}{\longrightarrow} \cdots \longrightarrow \operatorname{Map}\left(\bar{G}^{\times n}, k G\right) \stackrel{\delta_{n}}{\longrightarrow} \cdots,
$$

where the differential is given by

$$
\delta_{0}(x)(g)=g x-x g \quad(\text { for } x \in k G \text { and } g \in \bar{G})
$$

and (for $\varphi: \bar{G}^{\times n} \longrightarrow k G$ and $g_{1}, \cdots, g_{n+1} \in \bar{G}$ )

$$
\begin{aligned}
\delta_{n}(\varphi)\left(g_{1}, \cdots, g_{n+1}\right)= & g_{1} \varphi\left(g_{2}, \cdots, g_{n+1}\right)+ \\
& \sum_{i=1}^{n}(-1)^{i} \varphi\left(g_{1}, \cdots, g_{i} g_{i+1}, \cdots, g_{n+1}\right)+(-1)^{n+1} \varphi\left(g_{1}, \cdots, g_{n}\right) g_{n+1} .
\end{aligned}
$$

We keep the following notations in Section 3: $X$ is a complete set of representatives of the conjugacy classes in the finite group $G$. For $x \in X, C_{x}=\left\{g x g^{-1} \mid g \in G\right\}$ is the conjugacy class corresponding to $x$ and $C_{G}(x)=\left\{g \in G \mid g x g^{-1}=x\right\}$ is the centralizer subgroup. Now take a conjugacy class $C_{x}$ and define

$$
\begin{gathered}
\mathcal{H}_{x}^{0}=k C_{x}, \text { and for } n \geq 1, \\
\mathcal{H}_{x}^{n}=\left\{\varphi: \bar{G}^{\times n} \longrightarrow k G \mid \varphi\left(g_{1}, \cdots, g_{n}\right) \in k\left[g_{1} \cdots g_{n} C_{x}\right] \subset k G, \forall g_{1}, \cdots, g_{n} \in \bar{G}\right\},
\end{gathered}
$$

where $g_{1} \cdots g_{n} C_{x}$ denotes the subset of $G$ by multiplying $g_{1} \cdots g_{n}$ on $C_{x}$ and $k\left[g_{1} \cdots g_{n} C_{x}\right]$ is the $k$-subspace of $k G$ generated by this set. Note that we have $g_{1} \cdots g_{n} C_{x}=C_{x} g_{1} \cdots g_{n}$ and $k\left[g_{1} \cdots g_{n} C_{x}\right]=k\left[C_{x} g_{1} \cdots g_{n}\right]$. Let $\mathcal{H}_{x}^{*}=\bigoplus_{n \geq 0} \mathcal{H}_{x}^{n}$. Cibils and Solotar (4, Page 20, Proof of the theorem]) observed that $\mathcal{H}_{x}^{*}$ is a subcomplex of $\mathcal{H}^{*}$ and $\mathcal{H}^{*}=\bigoplus_{x \in X} \mathcal{H}_{x}^{*}$.

Lemma 6.1. $\mathcal{H}_{x}^{*}$ is canonically isomorphic to the complex $\operatorname{Hom}_{k G}\left(\operatorname{Bar}_{*}(k G) \otimes_{k G} k, k C_{x}\right)$, which computes the group cohomology $H^{*}\left(G, k C_{x}\right)$ of $G$ with coefficients in $k C_{x}$, where $k C_{x}$ is a left $k G$ module under conjugation.

Proof We know from Section 3 that the complex $\operatorname{Hom}_{k G}\left(\operatorname{Bar}_{*}(k G) \otimes_{k G} k, k C_{x}\right)$ is identified as the following complex:

$$
0 \longrightarrow k C_{x} \stackrel{\delta_{0}}{\longrightarrow} \operatorname{Map}\left(\bar{G}, k C_{x}\right) \stackrel{\delta_{1}}{\longrightarrow} \cdots \longrightarrow \operatorname{Map}\left(\bar{G}^{\times n}, k C_{x}\right) \stackrel{\delta_{n}}{\longrightarrow} \cdots,
$$

where the differential is given by

$$
\delta_{0}(x)(g)=g x g^{-1}-x \quad\left(\text { for } x \in k C_{x} \text { and } g \in \bar{G}\right)
$$

and (for $\varphi: \bar{G}^{\times n} \longrightarrow k C_{x}$ and $g_{1}, \cdots, g_{n+1} \in \bar{G}$ )

$$
\begin{aligned}
\delta_{n}(\varphi)\left(g_{1}, \cdots, g_{n+1}\right)= & g_{1} \varphi\left(g_{2}, \cdots, g_{n+1}\right) g_{1}^{-1}+ \\
& \sum_{i=1}^{n}(-1)^{i} \varphi\left(g_{1}, \cdots, g_{i} g_{i+1}, \cdots, g_{n+1}\right)+(-1)^{n+1} \varphi\left(g_{1}, \cdots, g_{n}\right) .
\end{aligned}
$$


A direct computation shows that the following map is an isomorphism of complexes:

$$
\begin{gathered}
\mathcal{H}_{x}^{*} \longrightarrow \operatorname{Hom}_{k G}\left(\operatorname{Bar}_{*}(k G) \otimes_{k G} k, k C_{x}\right), \\
\left(\varphi_{1}: \bar{G}^{\times n} \longrightarrow k G\right) \longmapsto\left(\varphi_{2}: \bar{G}^{\times n} \longrightarrow k C_{x}\right), \quad \varphi_{2}\left(g_{1}, \cdots, g_{n}\right)=\varphi_{1}\left(g_{1}, \cdots, g_{n}\right) g_{n}^{-1} \cdots g_{1}^{-1} .
\end{gathered}
$$

Its inverse is given by

$$
\begin{gathered}
\operatorname{Hom}_{k G}\left(\operatorname{Bar}_{*}(k G) \otimes_{k G} k, k C_{x}\right) \longrightarrow \mathcal{H}_{x}^{*}, \\
\left(\varphi_{2}: \bar{G}^{\times n} \longrightarrow k C_{x}\right) \longmapsto\left(\varphi_{1}: \bar{G}^{\times n} \longrightarrow k G\right), \quad \varphi_{1}\left(g_{1}, \cdots, g_{n}\right)=\varphi_{2}\left(g_{1}, \cdots, g_{n}\right) g_{1} \cdots g_{n} .
\end{gathered}
$$

Passing to the cohomology, we have $H^{*}\left(\mathcal{H}_{x}^{*}\right) \simeq H^{*}\left(G, k C_{x}\right)$.

Remark 6.2. Since the first three steps of the previous section realize

$$
H H^{*}(k G) \simeq H^{*}\left(G,{ }_{c} k G\right) \simeq \oplus_{x \in X} H^{*}\left(C_{G}(x), k\right),
$$

these isomorphisms also give a decomposition of the complex $\mathcal{H}^{*}$, which computes $H H^{*}(k G)$. In fact, during these three steps, we establish the following isomorphisms of complexes

$$
\begin{aligned}
& \mathcal{H}^{*}=\operatorname{Hom}_{k(G \times G}\left(\operatorname{Bar}_{*}(k G), k G\right) \\
& \stackrel{(1)}{\simeq} \operatorname{Hom}_{k(G \times G)}\left(k(G \times G) \otimes_{k G} \operatorname{Bar}_{*}(k G) \otimes_{k G} k, k G\right) \\
& \stackrel{(2)}{\simeq} \operatorname{Hom}_{k G}\left(\operatorname{Bar}_{*}(k G) \otimes_{k G} k,{ }_{c} k G\right) \\
& \stackrel{(3)}{=} \\
& \bigoplus_{x \in X} \operatorname{Hom}_{k G}\left(\operatorname{Bar}_{*}(k G) \otimes_{k G} k,{ }_{c} k C_{x}\right) .
\end{aligned}
$$

So the complex $\operatorname{Hom}_{k G}\left(\operatorname{Bar}_{*}(k G) \otimes_{k G} k,{ }_{c} k C_{x}\right)$ is isomorphic to a subcomplex of $\mathcal{H}^{*}$ and we verify easily that this subcomplex is just the above defined $\mathcal{H}_{x}^{*}$. However, the isomorphism between these two complexes is as follows:

$$
\begin{gathered}
\mathcal{H}_{x}^{*} \longrightarrow \operatorname{Hom}_{k G}\left(\operatorname{Bar}_{*}(k G) \otimes_{k G} k, k C_{x}\right), \\
\left(\varphi_{1}: \bar{G}^{\times n} \longrightarrow k G\right) \longmapsto\left(\varphi_{2}: \bar{G}^{\times n} \longrightarrow k C_{x}\right), \quad \varphi_{2}\left(g_{1}, \cdots, g_{n}\right)=(-1)^{\frac{n(n+1)}{2}} g_{1} \cdots g_{n} \varphi_{1}\left(g_{n}^{-1}, \cdots, g_{1}^{-1}\right) .
\end{gathered}
$$

Its inverse is given by

$$
\begin{aligned}
& \operatorname{Hom}_{k G}\left(\operatorname{Bar}_{*}(k G) \otimes_{k G} k, k C_{x}\right) \longrightarrow \mathcal{H}_{x}^{*}, \\
&\left(\varphi_{2}: \bar{G}^{\times n} \longrightarrow k C_{x}\right) \longmapsto\left(\varphi_{1}: \bar{G}^{\times n} \longrightarrow k G\right), \quad \varphi_{1}\left(g_{1}, \cdots, g_{n}\right)=(-1)^{\frac{n(n+1)}{2}} g_{1} \cdots g_{n} \varphi_{1}\left(g_{n}^{-1}, \cdots, g_{1}^{-1}\right) .
\end{aligned}
$$

Note that this isomorphism differs from the one in Lemma 6.1 by an automorphism of the complex $\operatorname{Hom}_{k G}\left(\operatorname{Bar}_{*}(k G) \otimes_{k G} k,{ }_{c} k C_{x}\right)$, which sends $\varphi: \bar{G}^{\times n} \rightarrow k C_{x}$ to $\varphi^{\prime}: \bar{G}^{\times n} \rightarrow k C_{x}$ with

$$
\varphi^{\prime}\left(g_{1}, \cdots, g_{n}\right)=(-1)^{\frac{n(n+1)}{2}} g_{1} \cdots g_{n} \varphi\left(g_{n}^{-1}, \cdots, g_{1}^{-1}\right) g_{n}^{-1} \cdots g_{1}^{-1} \text {. }
$$

On the other hand, we have shown that the complex $\operatorname{Hom}_{k G}\left(\operatorname{Bar}_{*}(k G) \otimes_{k G} k, k C_{x}\right)$ is isomorphic to the complex $\operatorname{Hom}_{k C_{G}(x)}\left(\operatorname{Bar}_{*}\left(k C_{G}(x)\right) \otimes_{k C_{G}(x)} k, k\right)$, which computes the group cohomology $H^{*}\left(C_{G}(x), k\right)$ of the centralizer subgroup $C_{G}(x)$ with coefficients in the trivial module $k$. (cf. Section 3 , from the fourth step to the six step.) Therefore we get another realization to the additive decomposition:

Theorem 6.3. Let $k$ be a field and $G$ a finite group. Consider the additive decomposition of Hochschild cohomology ring of the group algebra $k G$ :

$$
H H^{*}(k G) \simeq \bigoplus_{x \in X} H^{*}\left(C_{G}(x), k\right)
$$

where $X$ is a set of representatives of conjugacy classes of elements of $G$ and $C_{G}(x)$ is the centralizer subgroup of $G$. We compute the Hochschild cohomology $H H^{*}(k G)=H^{*}\left(H_{o m}(G \times G)\left(\operatorname{Bar}_{*}(k G), k G\right)\right)$ by the classical normalized bar resolution, and we compute the group cohomology $H^{*}\left(C_{G}(x), k\right)$ by $H^{*}\left(\operatorname{Hom}_{k C_{G}(x)}\left(\operatorname{Bar}_{*}\left(k C_{G}(x)\right) \otimes_{k C_{G}(x)} k, k\right)\right)$. Then, we can realize an isomorphism in additive decomposition as follows:

$$
\begin{aligned}
& H H^{*}(k G) \stackrel{\sim}{\longrightarrow} \bigoplus_{x \in X} H^{*}\left(C_{G}(x), k\right), \\
& {\left[\varphi_{x}: \bar{G}^{\times n} \longrightarrow k G\right], \quad \varphi_{x} \in \mathcal{H}_{x}^{n} \longmapsto\left[\widehat{\varphi}_{x}:{\overline{C_{G}(x)}}^{\times n} \longrightarrow k\right],}
\end{aligned}
$$




$$
\widehat{\varphi}_{x}\left(h_{1}, \cdots, h_{n}\right)=a_{1, x}, \text { where } \varphi_{x}\left(h_{1}, \cdots, h_{n}\right) h_{n}^{-1} \cdots h_{1}^{-1}=\sum_{i=1}^{n_{x}} a_{i, x} x_{i} \in k C_{x} .
$$

In other word, $\widehat{\varphi}_{x}\left(h_{1}, \cdots, h_{n}\right)$ is just the coefficient of $x$ in $\varphi_{x}\left(h_{1}, \cdots, h_{n}\right) h_{n}^{-1} \cdots h_{1}^{-1} \in k C_{x}$. The inverse of the above isomorphism is given as follows:

$$
\begin{aligned}
& \bigoplus_{x \in X} H^{*}\left(C_{G}(x), k\right) \stackrel{\sim}{\longrightarrow} H H^{*}(k G), \\
& {\left[\widehat{\varphi}_{x}:{\overline{C_{G}(x)}}^{\times n} \longrightarrow k\right] \longmapsto\left[\varphi_{x}: \bar{G}^{\times n} \longrightarrow k G\right], \quad \varphi_{x} \in \mathcal{H}_{x}^{n} \text {, }} \\
& \varphi_{x}\left(g_{1}, \cdots, g_{n}\right)=\sum_{i=1}^{n_{x}} \widehat{\varphi}_{x}\left(h_{i, 1}, \cdots, h_{i, n}\right) x_{i} g_{1} \cdots g_{n},
\end{aligned}
$$

where $h_{i, 1}, \cdots, h_{i, n} \in \overline{C_{G}(x)}$ are determined by the sequence $\left\{g_{1}, \cdots, g_{n}\right\}$ as follows:

$$
\gamma_{i, x} g_{1}=h_{i, 1} \gamma_{s_{i}^{1}, x}, \quad \gamma_{s_{i}^{1}, x} g_{2}=h_{i, 2} \gamma_{s_{i}^{2}, x}, \quad \cdots, \quad \gamma_{s_{i}^{n-1}, x} g_{n}=h_{i, n} \gamma_{s_{i}^{n}, x}
$$

Proof This is a combination of Lemma 6.1 and the correspondence from the fourth step to the six step in Section 3 .

By Remark 6.2, the two realizations of the additive decomposition in Theorem 5.1 and Theorem 6.3 are essentially the same on the cohomology group level. In the sequel, we prefer to the second realization since it is simpler.

\section{The CUP PRODUCT FORMULA}

We keep the notations of the previous sections: $k$ is a field, and $G$ is a finite group, and so on. We describe the cup product formula for the Hochschild cohomology $\operatorname{ring} H H^{*}(k G)$ in terms of the additive decomposition.

We shall define a product over $\sum_{x \in X} H^{*}\left(C_{G}(x), k\right)$ such that the isomorphism

$$
H H^{*}(k G) \simeq \bigoplus_{x \in X} H^{*}\left(C_{G}(x), k\right)
$$

realized in Theorem 6.3 becomes an isomorphism of graded algebras.

Let $\left[\widehat{\varphi_{x}}\right] \in H^{*}\left(C_{G}(x), k\right)$ (respectively $\left[\widehat{\varphi_{y}}\right] \in H^{m}\left(C_{G}(y), k\right)$ ) represented by the map $\widehat{\varphi_{x}}$ : ${\overline{C_{G}(x)}}^{\times n} \longrightarrow k$ (respectively by $\widehat{\varphi_{y}}:{\overline{C_{G}(y)}}^{\times m} \longrightarrow k$ ). Define

$$
\left[\widehat{\varphi_{x}} \cup \widehat{\varphi_{y}}\right]=\sum_{z \in X}\left[\left(\widehat{\varphi_{x}} \cup \widehat{\varphi_{y}}\right)_{z}\right] \in \bigoplus_{z \in X} H^{*}\left(C_{G}(z), k\right)
$$

with $\left(\widehat{\varphi_{x}} \cup \widehat{\varphi_{y}}\right)_{z}:{\overline{C_{G}(z)}}^{\times n+m} \rightarrow k$ as follows:

$$
\left(\widehat{\varphi_{x}} \cup \widehat{\varphi_{y}}\right)_{z}\left(h_{1}, \cdots, h_{n}, h_{n+1}, \cdots, h_{n+m}\right)=\sum_{(i, j) \in I_{1}^{\prime}} \widehat{\varphi_{x}}\left(h_{i, 1}, \cdots, h_{i, n}\right) \widehat{\varphi_{y}}\left(h_{j, 1}, \cdots, h_{j, m}\right),
$$

where

- $I_{1}^{\prime}$ is the set of pairs $(i, j)$ such that

$$
x_{i} h_{1} \cdots h_{n} y_{j}\left(h_{1} \cdots h_{n}\right)^{-1}=z
$$

-

$$
\gamma_{i, x} h_{1}=h_{i, 1} \gamma_{s_{i}^{1}, x}, \quad \gamma_{s_{i}^{1}, x} h_{2}=h_{i, 2} \gamma_{s_{i}^{2}, x}, \quad \cdots, \quad \gamma_{s_{i}^{n-1}, x} h_{n}=h_{i, n} \gamma_{s_{i}^{n}, x}
$$

$$
\gamma_{j, y} h_{n+1}=h_{j, 1} \gamma_{s_{j}^{1}, y}, \quad \gamma_{s_{j}^{1}, y} h_{n+2}=h_{j, 2} \gamma_{s_{j}^{2}, y}, \quad \cdots, \quad \gamma_{s_{j}^{m-1}, j} h_{n+m}=h_{j, m} \gamma_{s_{j}^{m}, y}
$$

Theorem 7.1. With the product defined above, the isomorphism in Theorem 6.3 is an isomorphism of graded algebras. 
Proof We shall show that with respect to the isomorphism in Theorem 6.3, the product defined above coincide with the cup product on the cohomology complex level.

Let $\left[\widehat{\varphi_{x}}\right]\left(\widehat{\varphi_{x}}:{\overline{C_{G}(x)}}^{\times n} \longrightarrow k\right)$ and $\left[\widehat{\varphi_{y}}\right]\left(\widehat{\varphi_{y}}:{\overline{C_{G}}(y)}^{\times m} \longrightarrow k\right)$ be two elements in $H^{n}\left(C_{G}(x), k\right)$ and in $H^{m}\left(C_{G}(y), k\right)$, respectively. Denote by $\left[\varphi_{x}: \bar{G}^{\times n} \longrightarrow k G\right]\left(\varphi_{x} \in \mathcal{H}_{x}^{n}\right)$ and $\left[\varphi_{y}: \bar{G}^{\times m} \longrightarrow\right.$ $k G]\left(\varphi_{y} \in \mathcal{H}_{y}^{m}\right)$ be the corresponding elements in $H H^{*}(k G)$. By Theorem 6.3.

$$
\begin{aligned}
\varphi_{x} & : \quad \bar{G}^{\times n} \longrightarrow k G, \\
\left(g_{1}, \cdots, g_{n}\right) & \mapsto \sum_{i=1}^{n_{x}} \widehat{\varphi_{x}}\left(h_{i, 1}, \cdots, h_{i, n}\right) x_{i} g_{1} \cdots g_{n},
\end{aligned}
$$

with

$$
\gamma_{i, x} g_{1}=h_{i, 1} \gamma_{s_{i}^{1}, x}, \quad \gamma_{s_{i}^{1}, x} g_{2}=h_{i, 2} \gamma_{s_{i}^{2}, x}, \quad \cdots, \quad \gamma_{s_{i}^{n-1}, x} g_{n}=h_{i, n} \gamma_{s_{i}^{n}, x} .
$$

A similar formula for $\varphi_{y}$ works as well.

Now denote by $\varphi_{x} \cup \varphi_{y}: \bar{G}^{\times(n+m)} \longrightarrow k G$ the cup product. By the definition of the cup product, for any $z \in X$, we obtain $\left(\varphi_{x} \cup \varphi_{y}\right)_{z} \in \mathcal{H}_{z}^{n+m}$ given by

$$
\begin{aligned}
\left(\varphi_{x} \cup \varphi_{y}\right)_{z} & : \quad \bar{G}^{\times(n+m)} \longrightarrow k G, \\
\left(g_{1}, \cdots, g_{n}, \cdots, g_{n+m}\right) & \mapsto \quad \sum_{k=1}^{n_{z}} \sum_{(i, j) \in I_{k}} \widehat{\varphi_{x}}\left(h_{i, 1}, \cdots, h_{i, n}\right) \widehat{\varphi_{y}}\left(h_{j, 1}, \cdots, h_{j, m}\right) z_{k} g_{1} \cdots g_{n+m},
\end{aligned}
$$

where

- $I_{k}$ is the set of pairs $(i, j)$ such that

$$
x_{i} g_{1} \cdots g_{n} y_{j}\left(g_{1} \cdots g_{n}\right)^{-1}=z_{k}
$$

-

$$
\gamma_{i, x} g_{1}=h_{i, 1} \gamma_{s_{i}^{1}, x}, \quad \gamma_{s_{i}^{1}, x} g_{2}=h_{i, 2} \gamma_{s_{i}^{2}, x}, \quad \cdots, \quad \gamma_{s_{i}^{n-1}, x} g_{n}=h_{i, n} \gamma_{s_{i}^{n}, x}
$$

$$
\gamma_{j, y} g_{n+1}=h_{j, 1} \gamma_{s_{j}^{1}, y}, \quad \gamma_{s_{j}^{1}, y} g_{n+2}=h_{j, 2} \gamma_{s_{j}^{2}, y}, \quad \cdots, \quad \gamma_{s_{j}^{m-1}, j} g_{n+m}=h_{j, m} \gamma_{s_{j}^{m}, y} .
$$

Note that $I_{k}$ depends on the elements $g_{1}, \cdots, g_{n}$. Again by Theorem 6.3 we obtain an element in $H^{*}\left(C_{G}(z), k\right)$ of the following form:

$$
\begin{aligned}
\overline{C_{G}(z)} \times(n+m) & \longrightarrow k \\
\left(h_{1}, \cdots, h_{n}, h_{n+1}, \cdots, h_{n+m}\right) & \longmapsto \sum_{(i, j) \in I_{1}} \widehat{\varphi_{x}}\left(h_{i, 1}, \cdots, h_{i, n}\right) \widehat{\varphi_{y}}\left(h_{j, 1}, \cdots, h_{j, m}\right),
\end{aligned}
$$

which is just $\left(\widehat{\varphi_{x}} \cup \widehat{\varphi_{y}}\right)_{z}$ defined before.

Similarly we can prove the following result.

Theorem 7.2. The isomorphism in Theorem 5.1 is an isomorphism of graded algebras with respect to the following product defined on $\bigoplus_{x \in X} H^{m}\left(C_{G}(x), k\right)$.

Let $\left[\widehat{\varphi}_{x}\right]\left(\widehat{\varphi}_{x}:{\overline{C_{G}(x)}}^{\times n} \longrightarrow k\right)$ and $\left[\widehat{\varphi}_{y}\right]\left(\widehat{\varphi}_{y}:{\overline{C_{G}(y)}}^{\times m} \longrightarrow k\right)$ be two elements in $H^{n}\left(C_{G}(x), k\right)$ and in $H^{m}\left(C_{G}(y), k\right)$, respectively. Define

$$
\left[\widehat{\varphi_{x}} \cup \widehat{\varphi_{y}}\right]=\sum_{z \in X}\left[\left(\widehat{\varphi_{x}} \cup \widehat{\varphi_{y}}\right)_{z}\right] \in \bigoplus_{z \in X} H^{*}\left(C_{G}(z), k\right)
$$

with $\left(\widehat{\varphi_{x}} \cup \widehat{\varphi_{y}}\right)_{z}:{\overline{C_{G}(z)}}^{\times n+m} \longrightarrow k$ as follows:

$$
\left(\widehat{\varphi_{x}} \cup \widehat{\varphi_{y}}\right)_{z}\left(h_{1}, \cdots, h_{n}, h_{n+1}, \cdots, h_{n+m}\right)=(-1)^{n m} \sum_{(i, j) \in I_{1}} \widehat{\varphi_{x}}\left(h_{i, 1}^{\prime}, \cdots, h_{i, n}^{\prime}\right) \widehat{\varphi_{y}}\left(h_{j, 1}^{\prime}, \cdots, h_{j, m}^{\prime}\right),
$$

where

- $I_{1}$ is the set of pairs $(i, j)$ such that

$$
h_{1} \cdots h_{m} x_{i}\left(h_{1} \cdots h_{m}\right)^{-1} y_{j}=z_{1}=z
$$

$$
\gamma_{i, x} h_{m+1}=h_{i, 1}^{\prime} \gamma_{s_{i}^{1}, x}, \quad \gamma_{s_{i}^{1}, x} h_{m+2}=h_{i, 2}^{\prime} \gamma_{s_{i}^{2}, x}, \quad \cdots, \quad \gamma_{s_{i}^{n-1}, x} h_{n+m}=h_{i, n}^{\prime} \gamma_{s_{i}^{n}, x}
$$


-

$$
\gamma_{j, y} h_{1}=h_{j, 1}^{\prime} \gamma_{s_{j}^{1}, y}, \quad \gamma_{s_{j}^{1}, y} h_{2}=h_{j, 2}^{\prime} \gamma_{s_{j}^{2}, y}, \quad \cdots, \quad \gamma_{s_{j}^{m-1}, j} h_{m}=h_{j, m}^{\prime} \gamma_{s_{j}^{m}, y} .
$$

Remark 7.3. (1) By Remark 5.2 (a), our cup product formulae in Theorems 7.1 and 7.2 are consistent with Siegel and Witherspoon's formula in [16. Theorem 5.1] up to an isomorphism.

(2) From our realization of the graded algebra isomorphism

$$
H H^{*}(k G) \simeq \bigoplus_{x \in X} H^{*}\left(C_{G}(x), k\right)=H^{*}(G, k) \oplus\left(\bigoplus_{x \in X-\{1\}} H^{*}\left(C_{G}(x), k\right)\right),
$$

it is clear that $H^{*}(G, k)$ can be seen as a graded subalgebra of $H H^{*}(k G)$ and each $H^{*}\left(C_{G}(x), k\right)$ is a graded $H^{*}(G, k)$-submodule of $H H^{*}(k G)$. Therefore, the additive decomposition gives an isomorphism of graded $H^{*}(G, k)$-modules.

\section{THE $\triangle$ OPERATOR FORMULA}

Let $k$ be a field and $G$ a finite group. Recall that the group algebra $k G$ is a symmetric algebra with the bilinear form

$$
\begin{gathered}
\langle,\rangle: k G \times k G \longrightarrow k, \\
\langle g, h\rangle= \begin{cases}1 & \text { if } g=h^{-1} \\
0 & \text { otherwise }\end{cases}
\end{gathered}
$$

for $g, h \in G$. For $n \geq 1$, the operator $\triangle: H H^{n}(k G) \longrightarrow H H^{n-1}(k G)$ on the Hochschild cohomology is defined by the equation

$$
\left\langle\triangle(\varphi)\left(g_{1}, \cdots, g_{n-1}\right), g_{n}\right\rangle=\sum_{i=1}^{n}(-1)^{i(n-1)}\left\langle\varphi\left(g_{i}, \cdots, g_{n-1}, g_{n}, g_{1}, \cdots, g_{i-1}\right), 1\right\rangle,
$$

where $\varphi \in C^{n}(k G) \simeq \operatorname{Map}\left(\bar{G}^{\times n}, k G\right), \triangle(\varphi) \in C^{n-1}(k G) \simeq M a p\left(\bar{G}^{\times n-1}, k G\right)$. Equivalently,

$$
\triangle(\varphi)\left(g_{1}, \cdots, g_{n-1}\right)=\sum_{g_{n} \in G} \sum_{i=1}^{n}(-1)^{i(n-1)}\left\langle\varphi\left(g_{i}, \cdots, g_{n-1}, g_{n}, g_{1}, \cdots, g_{i-1}\right), 1\right\rangle g_{n}^{-1} .
$$

This operator together with the cup product $\cup$ and the Lie bracket $[$,$] defines a BV algebra structure$ on $H H^{*}(k G)$.

We know from Section 4 that, for a conjugacy class $C_{x}$ of $G, \mathcal{H}_{x}^{*}=\bigoplus_{n \geq 0} \mathcal{H}_{x}^{n}$ is a subcomplex of the Hochschild cohomology complex $\mathcal{H}^{*}$, where

$$
\mathcal{H}_{x}^{n}=\left\{\varphi: \bar{G}^{\times n} \longrightarrow k G \mid \varphi\left(g_{1}, \cdots, g_{n}\right) \in k\left[g_{1} \cdots g_{n} C_{x}\right] \subset k G, \forall g_{1}, \cdots, g_{n} \in \bar{G}\right\} .
$$

Lemma 8.1. The operator $\triangle: \mathcal{H}^{n} \longrightarrow \mathcal{H}^{n-1}$ restricts to $\triangle_{x}: \mathcal{H}_{x}^{n} \longrightarrow \mathcal{H}_{x}^{n-1}$ for each conjugacy class $C_{x}$.

Proof We need to show that $\triangle(\varphi) \in \mathcal{H}_{x}^{n-1}$ for each $\varphi \in \mathcal{H}_{x}^{n}$. Let $g_{1}, \cdots, g_{n-1} \in \bar{G}$. Since

$$
\triangle(\varphi)\left(g_{1}, \cdots, g_{n-1}\right)=\sum_{g_{n} \in G} \sum_{i=1}^{n}(-1)^{i(n-1)}\left\langle\varphi\left(g_{i}, \cdots, g_{n-1}, g_{n}, g_{1}, \cdots, g_{i-1}\right), 1\right\rangle g_{n}^{-1},
$$

it suffices to prove the following statement: if $\left\langle\varphi\left(g_{i}, \cdots, g_{n-1}, g_{n}, g_{1}, \cdots, g_{i-1}\right), 1\right\rangle \neq 0$ for some $i$, then $g_{n}^{-1} \in g_{1} \cdots g_{n-1} C_{x}$. Indeed, $\left\langle\varphi\left(g_{i}, \cdots, g_{n-1}, g_{n}, g_{1}, \cdots, g_{i-1}\right), 1\right\rangle \neq 0$ implies that $1 \in$ $g_{i} \cdots g_{n-1} g_{n} g_{1} \cdots g_{i-1} C_{x}$, or equivalently, $g_{n}^{-1} \in g_{1} \cdots g_{i-1} C_{x} g_{i} \cdots g_{n-1}=g_{1} \cdots g_{n-1} C_{x}$.

Now we can determine the behavior of the operator $\triangle$ under the additive decomposition.

Theorem 8.2. Let $\widehat{\triangle}_{x}: H^{n}\left(C_{G}(x), k\right) \longrightarrow H^{n-1}\left(C_{G}(x), k\right)$ be the map induced by the operator $\triangle_{x}: H H^{n}(k G) \longrightarrow H H^{n-1}(k G)$ via the isomorphism established in Lemma 6.1. Then $\widehat{\triangle}_{x}$ is defined as follows:

$$
\widehat{\triangle}_{x}(\psi)\left(h_{1}, \cdots, h_{n-1}\right)=\sum_{i=1}^{n}(-1)^{i(n-1)} \psi\left(h_{i}, \cdots, h_{n-1}, h_{n-1}^{-1} \cdots h_{1}^{-1} x^{-1}, h_{1}, \cdots, h_{i-1}\right)
$$


for $\psi:{\overline{C_{G}(x)}}^{\times n} \longrightarrow k$ and for $h_{1}, \cdots, h_{n-1} \in \overline{C_{G}(x)}$.

Proof We shall prove that the following diagram

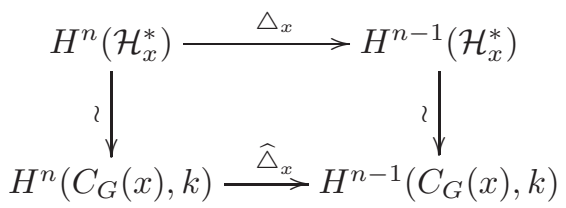

is commutative, where the vertical isomorphisms are given in Lemma 6.1.

Take an element $\psi:{\overline{C_{G}(x)}}^{\times n} \longrightarrow k$ in $\operatorname{Hom}_{k C_{G}(x)}\left(\operatorname{Bar}_{n}\left(k C_{G}(x)\right) \otimes_{k C_{G}(x)} k, k\right)$ and denote by $\varphi: \bar{G}^{\times n} \longrightarrow k G$ the corresponding element in $\mathcal{H}_{x}^{n}$. By Theorem 6.3. for any $h_{1}, \cdots, h_{n} \in \overline{C_{G}(x)}$, $\psi\left(h_{1}, \cdots, h_{n}\right)$ is equal to the coefficient of $x$ in $\varphi\left(h_{1}, \cdots, h_{n}\right) h_{n}^{-1} \cdots h_{1}^{-1} \in k C_{x}$. We should prove that $\widehat{\triangle}_{x}(\psi)$ corresponds to $\triangle_{x}(\varphi)$ via the isomorphism in Lemma 6.1

Now

$$
\triangle_{x}(\varphi)\left(g_{1}, \cdots, g_{n-1}\right)=\sum_{g_{n} \in G} \sum_{i=1}^{n}(-1)^{i(n-1)}\left\langle\varphi\left(g_{i}, \cdots, g_{n-1}, g_{n}, g_{1}, \cdots, g_{i-1}\right), 1\right\rangle g_{n}^{-1} .
$$

For any $h_{1}, \cdots, h_{n-1} \in \overline{C_{G}(x)}$, consider the coefficient of $x$ in $\triangle_{x}(\varphi)\left(h_{1}, \cdots, h_{n-1}\right) h_{n-1}^{-1} \cdots h_{1}^{-1} \in$ $k C_{x}$, or equivalently, the coefficient of $x h_{1} \cdots h_{n-1}$ in $\triangle_{x}(\varphi)\left(h_{1}, \cdots, h_{n-1}\right) \in k\left[h_{1} \cdots h_{n-1} C_{x}\right]$. This coefficient is equal to

$$
\begin{aligned}
& \left\langle\triangle_{x}(\varphi)\left(h_{1}, \cdots, h_{n-1}\right), h_{n-1}^{-1} \cdots h_{1}^{-1} x^{-1}\right\rangle \\
= & \sum_{i=1}^{n}(-1)^{i(n-1)}\left\langle\varphi\left(h_{i}, \cdots, h_{n-1}, h_{n-1}^{-1} \cdots h_{1}^{-1} x^{-1}, h_{1}, \cdots, h_{i-1}\right), 1\right\rangle .
\end{aligned}
$$

On the other hand, we also know that $\psi\left(h_{i}, \cdots, h_{n-1}, h_{n-1}^{-1} \cdots h_{1}^{-1} x^{-1}, h_{1}, \cdots, h_{i-1}\right)$ is equal to the coefficient of $x$ in

$$
\begin{aligned}
& \varphi\left(h_{i}, \cdots, h_{n-1}, h_{n-1}^{-1} \cdots h_{1}^{-1} x^{-1}, h_{1}, \cdots, h_{i-1}\right) h_{i-1}^{-1} \cdots h_{1}^{-1} x h_{1} \cdots h_{n-1} h_{n-1}^{-1} \cdots h_{i}^{-1} \\
= & \varphi\left(h_{i}, \cdots, h_{n-1}, h_{n-1}^{-1} \cdots h_{1}^{-1} x^{-1}, h_{1}, \cdots, h_{i-1}\right) x \in k C_{x},
\end{aligned}
$$

which is again equal to $\left\langle\varphi\left(h_{i}, \cdots, h_{n-1}, h_{n-1}^{-1} \cdots h_{1}^{-1} x^{-1}, h_{1}, \cdots, h_{i-1}\right), 1\right\rangle$. It follows that

$$
\widehat{\triangle}_{x}(\psi)\left(h_{1}, \cdots, h_{n-1}\right)=\sum_{i=1}^{n}(-1)^{i(n-1)} \psi\left(h_{i}, \cdots, h_{n-1}, h_{n-1}^{-1} \cdots h_{1}^{-1} x^{-1}, h_{1}, \cdots, h_{i-1}\right) .
$$

We have proved that $\widehat{\triangle}_{x}(\psi)$ corresponds to $\triangle_{x}(\varphi)$ via the isomorphism in Lemma 6.1 and the diagram is commutative (even at the cohomology complex level).

Remark 8.3. By [7, Corollary 2.2], we know that $H^{*}(G, k)$ is a Gerstenhaber subalgebra of $H H^{*}(k G)$ under the inclusion map:

$$
\begin{gathered}
\operatorname{Hom}_{k G}\left(\operatorname{Bar}_{*}(k G) \otimes_{k G} k, k\right) \hookrightarrow \operatorname{Hom}_{k(G \times G)}\left(\operatorname{Bar}_{*}(k G), k G\right), \\
\left(\varphi: \bar{G}^{\times n} \longrightarrow k\right) \longmapsto\left(\psi: \bar{G}^{\times n} \longrightarrow k G\right), \quad \psi\left(g_{1}, \cdots, g_{n}\right)=\varphi\left(g_{1}, \cdots, g_{n}\right) g_{1} \cdots g_{n},
\end{gathered}
$$

which is in fact induced by the isomorphism in Lemma 6.1 corresponding to $x=1$.

Notice that by notations in Section $6, \psi \in \mathcal{H}_{1}^{n}$. So motivated by Theorem 8.2 , we can similarly define an operator $\triangle_{1}: H^{n}(G, k) \longrightarrow H^{n-1}(G, k)$ in the group cohomology $H^{*}(G, k)$ as follows:

$$
\triangle_{1}(\varphi)\left(g_{1}, \cdots, g_{n-1}\right)=\sum_{i=1}^{n}(-1)^{i(n-1)} \varphi\left(g_{i}, \cdots, g_{n-1}, g_{n-1}^{-1} \cdots g_{1}^{-1}, g_{1}, \cdots, g_{i-1}\right)
$$

for $\varphi: \bar{G}^{\times n} \longrightarrow k$ and for $g_{1}, \cdots, g_{n-1} \in \bar{G}$.

We prove that $H^{*}(G, k)$ is in fact a BV subalgebra of $H H^{*}(k G)$.

Corollary 8.4. Let $k$ be a field and $G$ a finite group. Then $H^{*}(G, k) \hookrightarrow H H^{*}(k G)$ is a $B V$ subalgebra. 
Proof The inclusion in known to preserve the product structure. In fact this is a direct consequence of Theorem 7.1. In that result, taking $x=1=y$, we always has $z=1$ if the set $I_{1}^{\prime}$ is not empty.

As the inclusion $H^{n}(G, k) \hookrightarrow H H^{*}(k G)$ induced by the isomorphism in Lemma 6.1 corresponding to $x=1$, Theorem 8.2 shows that it preserves the $\triangle$-operator. Since this operator together with the cup product $\cup$ and the Lie bracket [, ] define a BV algebra structure on $H H^{*}(k G)$, via the isomorphism in Lemma 6.1] we deduce that the Lie bracket [, ] restricts to $H^{*}(G, k)=H^{*}\left(C_{G}(1), k\right)$.

Now we specialize to the case of abelian groups. Let $G$ be an abelian group. In this case, the Hochschild cohomology ring $H H^{*}(k G)$ of the group algebra $k G$ is isomorphic to the tensor product algebra of $k G$ and the group cohomology ring $H^{*}(G, k): H H^{*}(k G) \simeq k G \otimes_{k} H^{*}(G, k)$. According to [4, this isomorphism is given as follows. For $G$ an abelian group, conjugacy classes are elements of $G$, hence a cochain $\varphi_{x}$ of $\mathcal{H}_{x}^{n}$ for $x \in G$ attributes a scalar multiple of $g_{1} \cdots g_{n} x$ for each $\left(g_{1}, \cdots, g_{n}\right) \in \bar{G}^{\times n}$ and we obtain in this way a map $\overline{\varphi_{x}}: \bar{G}^{\times n} \longrightarrow k$. It is easy to see that the map $\widehat{\varphi_{x}}$ in Theorem 6.3 is just this scalar.

Now Theorem 7.1 shows that the map $\varphi \longmapsto \Sigma_{x \in G}\left(x \otimes \overline{\varphi_{x}}\right)$ defines a ring isomorphism $C^{*}(k G) \longrightarrow$ $k G \otimes C^{*}(k G, k)$ compatible with the differentials, and therefore it induces the above isomorphism. Theorem 8.2 specializes to the following statement.

Proposition 8.5. Let $k$ be a field and $G$ a finite abelian group. Under the above isomorphism $H H^{*}(k G) \simeq k G \otimes_{k} H^{*}(G, k)$, the operator $\triangle: H H^{n}(k G) \longrightarrow H H^{n-1}(k G)$ corresponds to the sum of operators $x \otimes \overline{\triangle_{x}}: x \otimes H^{n}(G, k) \longrightarrow x \otimes H^{n-1}(G, k)$, where $x \in G$ and $\overline{\triangle_{x}}: H^{n}(G, k) \longrightarrow H^{n-1}(G, k)$ is defined as follows:

$$
\bar{\triangle}_{x}(\bar{\varphi})\left(g_{1}, \cdots, g_{n-1}\right)=\sum_{i=1}^{n}(-1)^{i(n-1)} \bar{\varphi}\left(g_{i}, \cdots, g_{n-1}, g_{n-1}^{-1} \cdots g_{1}^{-1} x^{-1}, g_{1}, \cdots, g_{i-1}\right)
$$

for $\bar{\varphi}: \bar{G}^{\times n} \longrightarrow k$ and for $g_{1}, \cdots, g_{n-1} \in \bar{G}$.

Remark 8.6. We could also use the first realization to deduce a formula of the $\triangle$ operator. However, this formula is much more complicated than that of Theorem 8.2. We refrain from giving it here.

In a BV-algebra, we have the following equation (see 9]; Here we have changed the original equation according to the sign convention in Remark 3.1 and we omit the sign $\cup$ in the equation):

$$
\begin{aligned}
& \triangle(\alpha \beta \gamma)=(-1)^{|\alpha||\beta||\gamma|}\left[(-1)^{|\gamma|} \triangle(\alpha \beta) \gamma+\alpha \triangle(\beta \gamma)+(-1)^{|\alpha||\beta|} \beta \triangle(\alpha \gamma)\right. \\
&\left.-(-1)^{|\alpha|} \triangle(\alpha) \beta \gamma-(-1)^{|\alpha|+|\beta|-|\alpha||\gamma|} \alpha(\triangle(\beta)) \gamma-(-1)^{|\alpha|+|\beta|+|\gamma|} \alpha \beta \triangle(\gamma)\right],
\end{aligned}
$$

where $\alpha, \beta, \gamma \in H H^{*}(A)$ are homogeneous elements. So in order to compute the $\triangle$ operator in $H H^{*}(A)$, it suffices to find the value of $\triangle$ on each generator and on the cup product of every two generators. Finally, let us mention that we can use the cup product formula, the $\triangle$ operator formula and the following formula to compute the Lie bracket:

$$
[\alpha, \beta]=-(-1)^{(|\alpha|-1)|\beta|}\left(\triangle(\alpha \cup \beta)-\triangle(\alpha) \cup \beta-(-1)^{|\alpha|} \alpha \cup \triangle(\beta)\right) .
$$

\section{The SYMmetric GROUP OF DEGREe 3}

There are a few computations in literature on the BV structures of the Hochschild cohomology rings of some commutative algebras, see for example, 19. As far as we know, there is no concrete computation in non-commutative case. In this section, we use our method to compute the BV structure of the Hochschild cohomology rings of the group algebra $\mathbb{F}_{3} S_{3}$. The associative ring structure has been determined by Siegel and Witherspoon [16] using their cup product formula. So we only need to compute the $\triangle$ operator and the Lie bracket.

Let $G=S_{3}=\left\langle a, b \mid a^{3}=1=b^{2}, b a b=a^{-1}\right\rangle$. Choose the conjugacy class representatives as $1, a, b$. The corresponding centralizers are $H_{1}=G, H_{2}=\langle a\rangle$ and $H_{3}=\langle b\rangle$. So $H H^{*}\left(\mathbb{F}_{3} S_{3}\right) \simeq$ $H^{*}\left(S_{3}\right) \oplus H^{*}(\langle a\rangle) \oplus H^{*}(\langle b\rangle)$. The ring structures of $H^{*}\left(S_{3}\right)$, of $H^{*}(\langle a\rangle)$, and of $H^{*}(\langle b\rangle)$ are wellknown (see for example, [6]). $H^{*}\left(S_{3}\right)=\mathbb{F}_{3}[u, v] /\left(u^{2}\right)$, where $u$ and $v$ have degrees of 3 and 4 , respectively. $H^{*}(\langle a\rangle)=\mathbb{F}_{3}\left[w_{1}, w_{2}\right] /\left(w_{1}^{2}\right)$, where $w_{1}$ and $w_{2}$ have degrees of 1 and 2 , respectively. 
$H^{*}(\langle b\rangle)=\mathbb{F}_{3}$, since $\mathbb{F}_{3}\langle b\rangle$ is semisimple. Identify the elements $u, v$ with their images in $H H^{*}\left(\mathbb{F}_{3} S_{3}\right)$ and denote by $X_{1}, X_{2}$ the images of the elements (resp.) $w_{1}, w_{2}$ under the additive decomposition. Then Siegel and Witherspoon proved in [16] the following presentation for the Hochschild cohomology ring $H H^{*}\left(\mathbb{F}_{3} S_{3}\right): H H^{*}\left(\mathbb{F}_{3} S_{3}\right)$ is generated as an algebra by elements $u, v, C_{1}=1+a+a^{2}, C_{2}=$ $b\left(1+a+a^{2}\right), X_{1}, X_{2}$ of degrees (resp.) $3,4,0,0,1$ and 2 , subject to the relations

$$
\begin{gathered}
u X_{1}=0, \quad v X_{1}=u X_{2}, \quad u C_{2}=0=v C_{2}, \\
C_{i} X_{j}=0=C_{i} C_{j}(i, j \in\{1,2\}), \quad X_{1} X_{2}=u C_{1}, \quad X_{2}^{2}=v C_{1}
\end{gathered}
$$

in addition to the graded commutative relations.

Our formula in Theorem 8.2 for $\triangle$ operator is based on the normalized bar resolution. However, the real computations of the Hochschild cohomology or the group cohomology are based on the minimal projective resolutions. So we need to construct comparison maps between the minimal projective resolution and normalized bar resolution (by the same technique introduced in Section 2 ), and then we can transfer our formula in Theorem 8.2 to the minimal Hochschild cohomology level. By Theorem 8.2, the operator $\triangle: H H^{n}\left(\mathbb{F}_{3} S_{3}\right) \longrightarrow H H^{n-1}\left(\mathbb{F}_{3} S_{3}\right)$ restricts to the operators $\widehat{\triangle}_{b}: H^{n}(\langle b\rangle) \longrightarrow H^{n-1}(\langle b\rangle), \widehat{\triangle}_{a}: H^{n}(\langle a\rangle) \longrightarrow H^{n-1}(\langle a\rangle)$, and $\widehat{\triangle}_{1}: H^{n}\left(S_{3}\right) \longrightarrow H^{n-1}\left(S_{3}\right)$. Since $\mathbb{F}_{3}\langle b\rangle$ is semisimple and $H^{*}(\langle b\rangle)$ is concentrated in degree zero, $\widehat{\triangle}_{b}$ is trivial. $\mathbb{F}_{3}$ :

To compute $\widehat{\triangle}_{a}$, we first recall the minimal projective resolution $P_{a}^{*}$ of the trivial $\mathbb{F}_{3}\langle a\rangle$-module

$$
\cdots \longrightarrow \mathbb{F}_{3}\langle a\rangle \stackrel{a-1}{\longrightarrow} \mathbb{F}_{3}\langle a\rangle \stackrel{1+a+a^{2}}{\longrightarrow} \mathbb{F}_{3}\langle a\rangle \stackrel{a-1}{\longrightarrow} \mathbb{F}_{3}\langle a\rangle \stackrel{\epsilon}{\longrightarrow} \mathbb{F}_{3} \longrightarrow 0,
$$

where the differential $\epsilon$ is given by $\epsilon\left(\lambda_{1}+\lambda_{2} a+\lambda_{3} a^{2}\right)=\lambda_{1}+\lambda_{2}+\lambda_{3}$, and the differential $a-1$ means multiplying by $a-1$, etc.. There is a setwise self-homotopy over $P_{a}^{*}$ as follows:

$$
\begin{gathered}
t_{-1}: \mathbb{F}_{3} \rightarrow \mathbb{F}_{3}\langle a\rangle, \quad 1 \mapsto 1, \\
t_{0}: \mathbb{F}_{3}\langle a\rangle \rightarrow \mathbb{F}_{3}\langle a\rangle, \quad 1 \mapsto 0, a \mapsto 1, a^{2} \mapsto 1+a, \\
t_{1}: \mathbb{F}_{3}\langle a\rangle \rightarrow \mathbb{F}_{3}\langle a\rangle, \quad 1 \mapsto 0, a \mapsto 0, a^{2} \mapsto 1, \\
t_{2}: \mathbb{F}_{3}\langle a\rangle \rightarrow \mathbb{F}_{3}\langle a\rangle, \quad 1 \mapsto 0, a \mapsto 1, a^{2} \mapsto 1+a, \\
t_{3}: \mathbb{F}_{3}\langle a\rangle \rightarrow \mathbb{F}_{3}\langle a\rangle, \quad 1 \mapsto 0, a \mapsto 0, a^{2} \mapsto 1,
\end{gathered}
$$

We also have the normalized bar resolution $\operatorname{Bar}_{*}\left(\mathbb{F}_{3}\langle a\rangle\right) \otimes_{\mathbb{F}_{3}\langle a\rangle} \mathbb{F}_{3}$ of the trivial $\mathbb{F}_{3}\langle a\rangle$-module $\mathbb{F}_{3}$, which is identified as the following complex

$$
\cdots \longrightarrow \mathbb{F}_{3}\langle a\rangle \otimes{\overline{\mathbb{F}_{3}\langle a\rangle}}^{\otimes n} \stackrel{d_{n}}{\longrightarrow} \cdots \longrightarrow \mathbb{F}_{3}\langle a\rangle \otimes \overline{\mathbb{F}}_{3}\langle a\rangle \stackrel{d_{1}}{\longrightarrow} \mathbb{F}_{3}\langle a\rangle \stackrel{d_{0}}{\longrightarrow} \mathbb{F}_{3} \longrightarrow 0,
$$

where the differential is given by

$$
d_{0}\left(g_{0}\right)=1 \quad\left(\text { for } g_{0} \in\langle a\rangle\right)
$$

and (for $g_{0} \in\langle a\rangle, g_{1}, \cdots, g_{n} \in \overline{\langle a\rangle}$ )

$$
\begin{aligned}
& d_{n}\left(g_{0}, g_{1}, \cdots, g_{n}\right)=g_{0} g_{1} \otimes g_{2} \otimes \cdots \otimes g_{n}+ \\
& \qquad \sum_{i=1}^{n-1}(-1)^{i} g_{0} \otimes \cdots \otimes g_{i} g_{i+1} \otimes \cdots \otimes g_{n}+(-1)^{n} g_{0} \otimes g_{1} \otimes \cdots \otimes g_{n-1} .
\end{aligned}
$$

There is a setwise self-homotopy over $\operatorname{Bar}_{*}\left(\mathbb{F}_{3}\langle a\rangle\right) \otimes_{\mathbb{F}_{3}\langle a\rangle} \mathbb{F}_{3}$ as follows:

$$
\begin{aligned}
& s_{n}: \mathbb{F}_{3}\langle a\rangle \otimes{\overline{\mathbb{F}_{3}\langle a\rangle}}^{\otimes n} \longrightarrow \mathbb{F}_{3}\langle a\rangle \otimes{\overline{\mathbb{F}_{3}\langle a\rangle}}^{\otimes n+1}, \\
& g_{0} \otimes g_{1} \otimes \cdots \otimes g_{n} \longmapsto 1 \otimes g_{0} \otimes g_{1} \otimes \cdots \otimes g_{n},
\end{aligned}
$$

where $g_{0} \in\langle a\rangle, g_{1}, \cdots, g_{n} \in \overline{\langle a\rangle}$. Using $\left\{s_{n}\right\}$ and $\left\{t_{n}\right\}$ we get comparison maps $\Phi: P_{a}^{*} \longrightarrow$ $\operatorname{Bar}_{*}\left(\mathbb{F}_{3}\langle a\rangle\right) \otimes_{\mathbb{F}_{3}\langle a\rangle} \mathbb{F}_{3}$ and $\Psi: \operatorname{Bar}_{*}\left(\mathbb{F}_{3}\langle a\rangle\right) \otimes_{\mathbb{F}_{3}\langle a\rangle} \mathbb{F}_{3} \longrightarrow P_{a}^{*}$. We write down the maps up to degree 4 explicitly:

$$
\begin{aligned}
& \Phi: P_{a}^{*} \longrightarrow \operatorname{Bar}_{*}\left(\mathbb{F}_{3}\langle a\rangle\right) \otimes_{\mathbb{F}_{3}\langle a\rangle} \mathbb{F}_{3} \\
& \Phi_{-1}=i d: \mathbb{F}_{3} \longrightarrow \mathbb{F}_{3} \text {, } \\
& \Phi_{0}=i d: \mathbb{F}_{3}\langle a\rangle \longrightarrow \mathbb{F}_{3}\langle a\rangle, \\
& \Phi_{1}: \mathbb{F}_{3}\langle a\rangle \longrightarrow \mathbb{F}_{3}\langle a\rangle \otimes \overline{\mathbb{F}_{3}\langle a\rangle}, \quad g \mapsto g \otimes a, \text { for } g=1, a, a^{2},
\end{aligned}
$$




$$
\begin{aligned}
& \Phi_{2}: \mathbb{F}_{3}\langle a\rangle \longrightarrow \mathbb{F}_{3}\langle a\rangle \otimes{\overline{\mathbb{F}_{3}\langle a\rangle}}^{\otimes 2}, \quad g \mapsto g \otimes a \otimes a+g \otimes a^{2} \otimes a, \text { for } g=1, a, a^{2}, \\
& \Phi_{3}: \mathbb{F}_{3}\langle a\rangle \longrightarrow \mathbb{F}_{3}\langle a\rangle \otimes{\overline{F_{3}\langle a\rangle}}^{\otimes 3}, \quad g \mapsto g \otimes a \otimes a \otimes a+g \otimes a \otimes a^{2} \otimes a, \text { for } g=1, a, a^{2}, \\
& \Phi_{4}: \mathbb{F}_{3}\langle a\rangle \longrightarrow \mathbb{F}_{3}\langle a\rangle \otimes \overline{\mathbb{F}_{3}\langle a\rangle}{ }^{\otimes 4}, \quad g \mapsto g \otimes a \otimes a \otimes a \otimes a+g \otimes a \otimes a \otimes a^{2} \otimes a+ \\
& g \otimes a^{2} \otimes a \otimes a \otimes a+g \otimes a^{2} \otimes a \otimes a^{2} \otimes a, \text { for } g=1, a, a^{2} ; \\
& \Psi: \operatorname{Bar}_{*}\left(\mathbb{F}_{3}\langle a\rangle\right) \otimes_{\mathbb{F}_{3}\langle a\rangle} \mathbb{F}_{3} \longrightarrow P_{a}^{*} \\
& \Psi_{-1}=i d: \mathbb{F}_{3} \longrightarrow \mathbb{F}_{3} \text {, } \\
& \Psi_{0}=i d: \mathbb{F}_{3}\langle a\rangle \longrightarrow \mathbb{F}_{3}\langle a\rangle, \\
& \Psi_{1}: \mathbb{F}_{3}\langle a\rangle \otimes \overline{\mathbb{F}_{3}\langle a\rangle} \longrightarrow \mathbb{F}_{3}\langle a\rangle, \quad g \otimes a \mapsto g, \quad g \otimes a^{2} \mapsto g(1+a), \text { for } g=1, a, a^{2}, \\
& \Psi_{2}: \mathbb{F}_{3}\langle a\rangle \otimes{\overline{\mathbb{F}_{3}\langle a\rangle}}^{\otimes 2} \longrightarrow \mathbb{F}_{3}\langle a\rangle, \quad g \otimes a \otimes a \mapsto 0, \\
& g \otimes a \otimes a^{2} \mapsto g, \quad g \otimes a^{2} \otimes a \mapsto g, \quad g \otimes a^{2} \otimes a^{2} \mapsto g, \text { for } g=1, a, a^{2}, \\
& \Psi_{3}: \mathbb{F}_{3}\langle a\rangle \otimes{\overline{\mathbb{F}_{3}\langle a\rangle}}^{\otimes 3} \longrightarrow \mathbb{F}_{3}\langle a\rangle, \quad g \otimes a \otimes a \otimes a \mapsto 0, \quad g \otimes a \otimes a \otimes a^{2} \mapsto 0, \\
& g \otimes a \otimes a^{2} \otimes a \mapsto 0, \quad g \otimes a \otimes a^{2} \otimes a^{2} \mapsto 0, \quad g \otimes a^{2} \otimes a \otimes a \mapsto 0,
\end{aligned}
$$

$g \otimes a^{2} \otimes a \otimes a^{2} \mapsto g(1+a), \quad g \otimes a^{2} \otimes a^{2} \otimes a \mapsto g(1+a), \quad g \otimes a^{2} \otimes a^{2} \otimes a^{2} \mapsto g a$, for $g=1, a, a^{2}$,

$$
\begin{gathered}
\Psi_{4}: \mathbb{F}_{3}\langle a\rangle \otimes \overline{\mathbb{F}}_{3}\langle a\rangle \\
g \otimes a \otimes \mathbb{F}_{3}\langle a\rangle, \quad g \otimes a \otimes a \otimes a \otimes a \mapsto 0, \quad g \otimes a \otimes a \otimes a \otimes a^{2} \mapsto 0, \\
g \otimes a \otimes a^{2} \otimes a \otimes a^{2} \mapsto a \mapsto 0, \quad g \otimes a \otimes a \otimes a^{2} \otimes a^{2} \mapsto 0, \quad g \otimes a \otimes a^{2} \otimes a \otimes a \mapsto 0, \\
g \otimes a^{2} \otimes a \otimes a \otimes a \mapsto 0, \quad g \otimes a \otimes a^{2} \otimes a^{2} \otimes a \mapsto g, \quad g \otimes a \otimes a^{2} \otimes a^{2} \otimes a^{2} \mapsto g, \\
g \otimes a^{2} \otimes a \otimes a^{2} \otimes a^{2} \mapsto 0, \quad g \otimes a^{2} \otimes a^{2} \otimes a \otimes a \mapsto 0, \quad g \otimes a^{2} \mapsto 0, \quad g \otimes a^{2} \otimes a \otimes a^{2} \otimes a \mapsto 0, \\
g \otimes a^{2} \otimes a \otimes a^{2} \otimes a^{2} \otimes a \mapsto g, \quad g \otimes a^{2} \otimes a^{2} \otimes a^{2} \otimes a^{2} \mapsto 0, \text { for } g=1, a, a^{2} .
\end{gathered}
$$

We have the following commutative diagram:

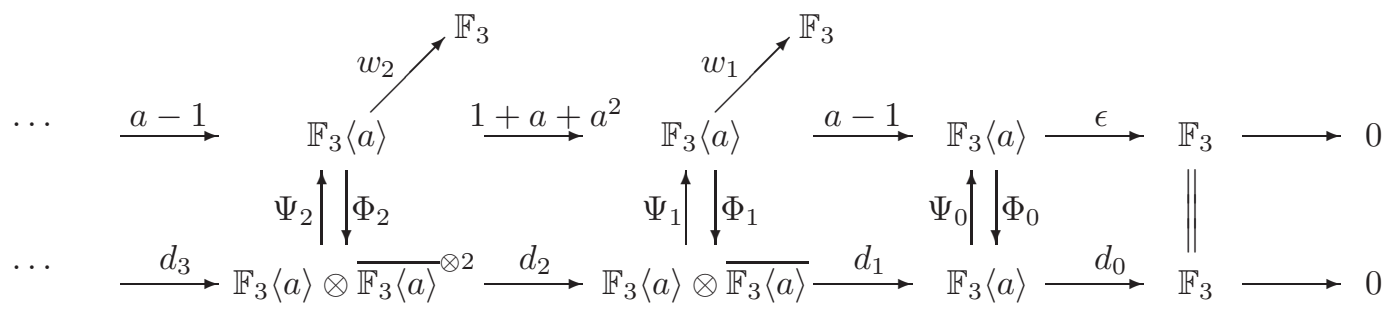

Clearly both the representatives of $w_{1}$ and $w_{2}$ in the group cohomology $H^{*}(\langle a\rangle)=\mathbb{F}_{3}\left[w_{1}, w_{2}\right] /\left(w_{1}^{2}\right)$ can be chosen as $\epsilon: \mathbb{F}_{3}\langle a\rangle \longrightarrow \mathbb{F}_{3}, \lambda_{1}+\lambda_{2} a+\lambda_{3} a^{2} \mapsto \lambda_{1}+\lambda_{2}+\lambda_{3}$. By abuse of notation, we have

$$
\begin{aligned}
& \widehat{\triangle}_{a}\left(w_{1}\right)=\widehat{\triangle}_{a}\left(w_{1} \Psi_{1}\right) \circ \Phi_{0}, \\
& \widehat{\triangle}_{a}\left(w_{2}\right)=\widehat{\triangle}_{a}\left(w_{2} \Psi_{2}\right) \circ \Phi_{1} .
\end{aligned}
$$

A straightforward calculation shows that $\widehat{\triangle}_{a}\left(w_{1}\right)=-1$ and $\widehat{\triangle}_{a}\left(w_{2}\right)=0$. Similarly, we can get that

$$
\begin{gathered}
\widehat{\triangle}_{a}\left(w_{1} w_{2}\right)=\widehat{\triangle}_{a}\left(\left(w_{1} \Psi_{1}\right)\left(w_{2} \Psi_{2}\right)\right) \circ \Phi_{2}, \\
\widehat{\triangle}_{a}\left(w_{2}^{2}\right)=\widehat{\triangle}_{a}\left(\left(w_{2} \Psi_{2}\right)^{2}\right) \circ \Phi_{3} .
\end{gathered}
$$

By direct computation, we have $\left(w_{1} \Psi_{1}\right)\left(w_{2} \Psi_{2}\right) \Phi_{3}(1)=1$ and $\left.\left(w_{2} \Psi_{2}\right)^{2}\right) \Phi_{4}(1)=1$, which imply that both the representatives of $w_{1} w_{2}$ and $w_{2}^{2}$ in the group cohomology $H^{*}(\langle a\rangle)=\mathbb{F}_{3}\left[w_{1}, w_{2}\right] /\left(w_{1}^{2}\right)$ are given by $\epsilon$. So again a straightforward calculation shows that $\widehat{\triangle}_{a}\left(w_{1} w_{2}\right)=-w_{2}$ and $\widehat{\triangle}_{a}\left(w_{2}^{2}\right)=0$.

Next we compute $\widehat{\triangle}_{1}$. First of all, we need to construct a minimal projective resolution $P_{1}^{*}$ of the trivial $\mathbb{F}_{3} S_{3}$-module $\mathbb{F}_{3}$. Recall that the group algebra $\mathbb{F}_{3} S_{3}$ can be identified as the $\mathbb{F}_{3}$-algebra $A$ given by the following quiver and relations:

$$
\stackrel{1}{\stackrel{\alpha}{\rightleftarrows}} \stackrel{2}{\circ}, \quad \alpha \beta \alpha=\beta \alpha \beta=0 .
$$


Let ${ }_{A} A=A e_{1} \oplus A e_{2}=\left\langle e_{1}, \alpha, \beta \alpha\right\rangle \oplus\left\langle e_{2}, \beta, \alpha \beta\right\rangle$ be the decomposition of the regular module into the indecomposable projective modules. Then we have the following (Remind that all the computations take place over $\left.\mathbb{F}_{3}\right)$ :

$$
\begin{gathered}
e_{1}=-(1+b), e_{2}=-(1-b), \\
\alpha=-a(1-a)(1+b)=-(1-b) a(1-a)=-\left(a-a^{2}+a b-a^{2} b\right), \\
\beta=-a(1-a)(1-b)=-(1+b) a(1-a)=-\left(a-a^{2}-a b+a^{2} b\right), \\
\beta \alpha=(1+b)\left(1+a+a^{2}\right)(1+b)=-\left(1+a+b+a^{2}+a b+a^{2} b\right), \\
\alpha \beta=(1-b)\left(1+a+a^{2}\right)(1-b)=-\left(1+a-b+a^{2}-a b-a^{2} b\right), \\
1=e_{1}+e_{2}, a=e_{1}+e_{2}-\alpha-\beta-\alpha \beta-\beta \alpha, b=e_{1}-e_{2}, \\
a^{2}=e_{1}+e_{2}+\alpha+\beta-\alpha \beta-\beta \alpha, a b=e_{1}-e_{2}-\alpha+\beta+\alpha \beta-\beta \alpha, \\
a^{2} b=e_{1}-e_{2}+\alpha-\beta+\alpha \beta-\beta \alpha .
\end{gathered}
$$

$A e_{1}$ is the projective cover of the trivial module $\mathbb{F}_{3}$ since $a$ and $b$ act trivially on $A e_{1} / \operatorname{rad}\left(A e_{1}\right) ; A e_{2}$ is the projective cover of the sign module sgn since $a$ (resp., $b$ ) acts trivially (resp., by multiplying -1) on $A e_{2} / \operatorname{rad}\left(A e_{2}\right)$. Now it is easy to write down the minimal projective resolution $P_{1}^{*}$ of the trivial $\mathbb{F}_{3} S_{3}$-module $\mathbb{F}_{3}$ is as follows:

$$
\cdots \longrightarrow A e_{1} \stackrel{\partial_{4}}{\longrightarrow} A e_{1} \stackrel{\partial_{3}}{\longrightarrow} A e_{2} \stackrel{\partial_{2}}{\longrightarrow} A e_{2} \stackrel{\partial_{1}}{\longrightarrow} A e_{1} \stackrel{\partial_{0}}{\longrightarrow} \mathbb{F}_{3} \longrightarrow 0,
$$

where the differential is given as follows:

$$
\begin{gathered}
\partial_{0}: e_{1} \mapsto 1, \alpha \mapsto 0, \beta \alpha \mapsto 0, \\
\partial_{1}: e_{2} \mapsto \alpha, \beta \mapsto \beta \alpha, \alpha \beta \mapsto 0, \\
\partial_{2}: e_{2} \mapsto \alpha \beta, \beta \mapsto 0, \alpha \beta \mapsto 0, \\
\partial_{3}: e_{1} \mapsto \beta, \alpha \mapsto \alpha \beta, \beta \alpha \mapsto 0, \\
\partial_{4}: e_{1} \mapsto \beta \alpha, \alpha \mapsto 0, \beta \alpha \mapsto 0, \\
\partial_{5}: e_{2} \mapsto \alpha, \beta \mapsto \beta \alpha, \alpha \beta \mapsto 0, \\
\partial_{6}: e_{2} \mapsto \alpha \beta, \beta \mapsto 0, \alpha \beta \mapsto 0, \\
\partial_{7}: e_{1} \mapsto \beta, \alpha \mapsto \alpha \beta, \beta \alpha \mapsto 0, \\
\partial_{8}: e_{1} \mapsto \beta \alpha, \alpha \mapsto 0, \beta \alpha \mapsto 0,
\end{gathered}
$$

Using the Lowey diagram structures of $A e_{1}$ and $A e_{2}$, we can easily construct a setwise self-homotopy over $P_{1}^{*}$ as follows:

$$
\begin{gathered}
t_{-1}: \mathbb{F}_{3} \rightarrow A e_{1}, \quad 1 \mapsto e_{1}, \\
t_{0}: A e_{1} \rightarrow A e_{2}, \quad e_{1} \mapsto 0, \alpha \mapsto e_{2}, \beta \alpha \mapsto \beta, \\
t_{1}: A e_{2} \rightarrow A e_{2}, \quad e_{2} \mapsto 0, \beta \mapsto 0, \alpha \beta \mapsto e_{2}, \\
t_{2}: A e_{2} \rightarrow A e_{1}, \quad e_{2} \mapsto 0, \beta \mapsto e_{1}, \alpha \beta \mapsto \alpha, \\
t_{3}: A e_{1} \rightarrow A e_{1}, \quad e_{1} \mapsto 0, \alpha \mapsto 0, \beta \alpha \mapsto e_{1}, \\
t_{4}: A e_{1} \rightarrow A e_{2}, \quad e_{1} \mapsto 0, \alpha \mapsto e_{2}, \beta \alpha \mapsto \beta, \\
t_{5}: A e_{2} \rightarrow A e_{2}, \quad e_{2} \mapsto 0, \beta \mapsto 0, \alpha \beta \mapsto e_{2}, \\
t_{6}: A e_{2} \rightarrow A e_{1}, \quad e_{2} \mapsto 0, \beta \mapsto e_{1}, \alpha \beta \mapsto \alpha, \\
t_{7}: A e_{1} \rightarrow A e_{1}, \quad e_{1} \mapsto 0, \alpha \mapsto 0, \beta \alpha \mapsto e_{1}, \\
t_{8}: A e_{1} \rightarrow A e_{2}, \quad e_{1} \mapsto 0, \alpha \mapsto e_{2}, \beta \alpha \mapsto \beta,
\end{gathered}
$$

We also have the normalized bar resolution $\operatorname{Bar}_{*}(A) \otimes_{A} \mathbb{F}_{3}$ of the trivial $A$-module $\mathbb{F}_{3}$, which is identified as the following complex

$$
\cdots \longrightarrow A \otimes \bar{A}^{\otimes n} \stackrel{d_{n}}{\longrightarrow} \cdots \longrightarrow A \otimes \bar{A} \stackrel{d_{1}}{\longrightarrow} A \stackrel{d_{0}}{\longrightarrow} \mathbb{F}_{3} \longrightarrow 0,
$$

where the differential is given by

$$
d_{0}\left(g_{0}\right)=1 \quad\left(\text { for } g_{0} \in S_{3}\right)
$$


and (for $g_{0} \in S_{3}, g_{1}, \cdots, g_{n} \in \overline{S_{3}}$ )

$$
\begin{aligned}
& d_{n}\left(g_{0}, g_{1}, \cdots, g_{n}\right)= g_{0} g_{1} \otimes g_{2} \otimes \cdots \otimes g_{n}+ \\
& \qquad \sum_{i=1}^{n-1}(-1)^{i} g_{0} \otimes \cdots \otimes g_{i} g_{i+1} \otimes \cdots \otimes g_{n}+(-1)^{n} g_{0} \otimes g_{1} \otimes \cdots \otimes g_{n-1} .
\end{aligned}
$$

There is a setwise self-homotopy over $\operatorname{Bar}_{*}(A) \otimes_{A} \mathbb{F}_{3}$ as follows:

$$
\begin{gathered}
s_{n}: A \otimes \bar{A}^{\otimes n} \longrightarrow A \otimes \bar{A}^{\otimes n+1}, \\
g_{0} \otimes g_{1} \otimes \cdots \otimes g_{n} \longmapsto 1 \otimes g_{0} \otimes g_{1} \otimes \cdots \otimes g_{n},
\end{gathered}
$$

where $g_{0} \in S_{3}, g_{1}, \cdots, g_{n} \in \overline{S_{3}}$. As before, we want to use $\left\{s_{n}\right\}$ and $\left\{t_{n}\right\}$ to get comparison maps $\Lambda: P_{1}^{*} \longrightarrow \operatorname{Bar}_{*}(A) \otimes_{A} \mathbb{F}_{3}$ and $\Theta: \operatorname{Bar}_{*}(A) \otimes_{A} \mathbb{F}_{3} \longrightarrow P_{1}^{*}$. Here the situation is a bit different, since $P_{1}^{*}$ is not a free resolution. However, if we replace $s_{n}(x)$ by $\widetilde{s_{n}}(x)=e_{1} s_{n}\left(e_{1} x\right)+e_{2} s_{n}\left(e_{2} x\right)$, then the method introduced in Section 2 still works. We write down the comparison maps up to degree 8 explicitly:

$$
\begin{gathered}
\Lambda: P_{1}^{*} \longrightarrow \operatorname{Bar}_{*}(A) \otimes_{A} \mathbb{F}_{3} \\
\Lambda_{-1}=i d: \mathbb{F}_{3} \longrightarrow \mathbb{F}_{3}, \\
\Lambda_{0}: A e_{1} \longrightarrow A, \\
a e_{1} \mapsto a e_{1}, \\
\Lambda_{1}: A e_{2} \longrightarrow A \otimes \bar{A}, \\
e_{2} \mapsto e_{2} \otimes \alpha, \beta \mapsto \beta \otimes \alpha, \alpha \beta \mapsto \alpha \beta \otimes \alpha, \\
\Lambda_{2}: A e_{2} \longrightarrow A \otimes \bar{A}^{\otimes 2}, \\
e_{2} \mapsto e_{2} \otimes \alpha \beta \otimes \alpha, \beta \mapsto \beta \otimes \alpha \beta \otimes \alpha, \alpha \beta \mapsto \alpha \beta \otimes \alpha \beta \otimes \alpha, \\
\Lambda_{3}: A e_{1} \longrightarrow A \otimes \bar{A}^{\otimes 3}, \\
\Lambda_{1} \mapsto A e_{1} \longrightarrow A \otimes e_{1} \otimes \beta \otimes \alpha \beta \otimes \alpha, \alpha \mapsto \alpha \otimes \beta \otimes \alpha \beta \otimes \alpha, \beta \alpha \mapsto \beta \alpha \otimes \beta \otimes \alpha \beta \otimes \alpha,
\end{gathered}
$$

$e_{1} \mapsto e_{1} \otimes \beta \alpha \otimes \beta \otimes \alpha \beta \otimes \alpha, \alpha \mapsto \alpha \otimes \beta \alpha \otimes \beta \otimes \alpha \beta \otimes \alpha, \beta \alpha \mapsto \beta \alpha \otimes \beta \alpha \otimes \beta \otimes \alpha \beta \otimes \alpha$,

$$
\begin{gathered}
\Lambda_{5}: A e_{2} \longrightarrow A \otimes \bar{A}^{\otimes 5}, \\
e_{2} \mapsto e_{2} \otimes \alpha \otimes \beta \alpha \otimes \beta \otimes \alpha \beta \otimes \alpha, \beta \mapsto \beta \otimes \alpha \otimes \beta \alpha \otimes \beta \otimes \alpha \beta \otimes \alpha,
\end{gathered}
$$$$
\alpha \beta \mapsto \alpha \beta \otimes \alpha \otimes \beta \alpha \otimes \beta \otimes \alpha \beta \otimes \alpha,
$$

$$
\Lambda_{6}: A e_{2} \longrightarrow A \otimes \bar{A}^{\otimes 6}
$$

$e_{2} \mapsto e_{2} \otimes \alpha \beta \otimes \alpha \otimes \beta \alpha \otimes \beta \otimes \alpha \beta \otimes \alpha, \beta \mapsto \beta \otimes \alpha \beta \otimes \alpha \otimes \beta \alpha \otimes \beta \otimes \alpha \beta \otimes \alpha$,

$$
\begin{gathered}
\alpha \beta \mapsto \alpha \beta \otimes \alpha \beta \otimes \alpha \otimes \beta \alpha \otimes \beta \otimes \alpha \beta \otimes \alpha, \\
\Lambda_{7}: A e_{1} \longrightarrow A \otimes \bar{A}^{\otimes 7},
\end{gathered}
$$

$e_{1} \mapsto e_{1} \otimes \beta \otimes \alpha \beta \otimes \alpha \otimes \beta \alpha \otimes \beta \otimes \alpha \beta \otimes \alpha, \alpha \mapsto \alpha \otimes \beta \otimes \alpha \beta \otimes \alpha \otimes \beta \alpha \otimes \beta \otimes \alpha \beta \otimes \alpha$,

$$
\beta \alpha \mapsto \beta \alpha \otimes \beta \otimes \alpha \beta \otimes \alpha \otimes \beta \alpha \otimes \beta \otimes \alpha \beta \otimes \alpha,
$$

$$
\Lambda_{8}: A e_{1} \longrightarrow A \otimes \bar{A}^{\otimes 8}
$$

$e_{1} \mapsto e_{1} \otimes \beta \alpha \otimes \beta \otimes \alpha \beta \otimes \alpha \otimes \beta \alpha \otimes \beta \otimes \alpha \beta \otimes \alpha, \alpha \mapsto \alpha \otimes \beta \alpha \otimes \beta \otimes \alpha \beta \otimes \alpha \otimes \beta \alpha \otimes \beta \otimes \alpha \beta \otimes \alpha$,

$$
\beta \alpha \mapsto \beta \alpha \otimes \beta \alpha \otimes \beta \otimes \alpha \beta \otimes \alpha \otimes \beta \alpha \otimes \beta \otimes \alpha \beta \otimes \alpha ;
$$

$$
\begin{gathered}
\Theta: \operatorname{Bar}_{*}(A) \otimes_{A} \mathbb{F}_{3} \longrightarrow P_{1}^{*} \\
\Theta_{-1}=i d: \mathbb{F}_{3} \longrightarrow \mathbb{F}_{3}, \\
\Theta_{0}: A \longrightarrow A e_{1}, \\
a \mapsto a e_{1}, \text { for } a \in A, \\
\Theta_{1}: A \otimes \bar{A} \longrightarrow A e_{2},
\end{gathered}
$$

$$
1 \otimes g_{1} \mapsto-e_{2}-\beta, 0, e_{2}-\beta,-e_{2}-\beta, e_{2}-\beta, \text { for } g_{1}=a, b, a^{2}, a b, a^{2} b,
$$$$
\Theta_{2}: A \otimes \bar{A}^{\otimes 2} \longrightarrow A e_{2},
$$

$1 \otimes g_{1} \otimes g_{2} \mapsto-e_{2}, 0,0,0, e_{2}$ for $g_{1}=a, b, a^{2}, a b, a^{2} b$,

and where $\Theta_{1}\left(1 \otimes g_{2}\right)=-e_{2}-\beta$, 
$1 \otimes g_{1} \otimes g_{2} \mapsto 0,0, e_{2},-e_{2}, 0$ for $g_{1}=a, b, a^{2}, a b, a^{2} b$,

and where $\Theta_{1}\left(1 \otimes g_{2}\right)=e_{2}-\beta$,

$1 \otimes g_{1} \otimes g_{2} \mapsto 0$, for any other case,

$$
\Theta_{3}: A \otimes \bar{A}^{\otimes 3} \longrightarrow A e_{1},
$$

$1 \otimes g_{1} \otimes g_{2} \otimes g_{3} \mapsto-e_{1}-\alpha, 0, e_{1}-\alpha, e_{1}+\alpha,-e_{1}+\alpha$, for $g_{1}=a, b, a^{2}, a b, a^{2} b$,

and where $\Theta_{2}\left(1 \otimes g_{2} \otimes g_{3}\right)=e_{2}$,

$1 \otimes g_{1} \otimes g_{2} \otimes g_{3} \mapsto e_{1}+\alpha, 0,-e_{1}+\alpha,-e_{1}-\alpha, e_{1}-\alpha$, for $g_{1}=a, b, a^{2}, a b, a^{2} b$,

and where $\Theta_{2}\left(1 \otimes g_{2} \otimes g_{3}\right)=-e_{2}$,

$1 \otimes g_{1} \otimes g_{2} \otimes g_{3} \mapsto 0$, for any other case,

$$
\Theta_{4}: A \otimes \bar{A}^{\otimes 4} \longrightarrow A e_{1}
$$

$1 \otimes g_{1} \otimes g_{2} \otimes g_{3} \otimes g_{4} \mapsto e_{1}, 0,0,0, e_{1}$ for $g_{1}=a, b, a^{2}, a b, a^{2} b$,

and where $\Theta_{3}\left(1 \otimes g_{2} \otimes g_{3} \otimes g_{4}\right)=e_{1}+\alpha$,

$1 \otimes g_{1} \otimes g_{2} \otimes g_{3} \otimes g_{4} \mapsto 0,0, e_{1}, e_{1}, 0$ for $g_{1}=a, b, a^{2}, a b, a^{2} b$,

and where $\Theta_{3}\left(1 \otimes g_{2} \otimes g_{3} \otimes g_{4}\right)=e_{1}-\alpha$,

$1 \otimes g_{1} \otimes g_{2} \otimes g_{3} \otimes g_{4} \mapsto-e_{1}, 0,0,0,-e_{1}$ for $g_{1}=a, b, a^{2}, a b, a^{2} b$,

and where $\Theta_{3}\left(1 \otimes g_{2} \otimes g_{3} \otimes g_{4}\right)=-e_{1}-\alpha$,

$1 \otimes g_{1} \otimes g_{2} \otimes g_{3} \otimes g_{4} \mapsto 0,0,-e_{1},-e_{1}, 0$ for $g_{1}=a, b, a^{2}, a b, a^{2} b$,

and where $\Theta_{3}\left(1 \otimes g_{2} \otimes g_{3} \otimes g_{4}\right)=-e_{1}+\alpha$,

$1 \otimes g_{1} \otimes g_{2} \otimes g_{3} \otimes g_{4} \mapsto 0$, for any other case,

$$
\Theta_{5}: A \otimes \bar{A}^{\otimes 5} \longrightarrow A e_{2},
$$

$1 \otimes g_{1} \otimes g_{2} \otimes g_{3} \otimes g_{4} \otimes g_{5} \mapsto-e_{2}-\beta, 0, e_{2}-\beta,-e_{2}-\beta, e_{2}-\beta$, for $g_{1}=a, b, a^{2}, a b, a^{2} b$,

and where $\Theta_{4}\left(1 \otimes g_{2} \otimes g_{3} \otimes g_{4} \otimes g_{5}\right)=e_{1}$,

$1 \otimes g_{1} \otimes g_{2} \otimes g_{3} \otimes g_{4} \otimes g_{5} \mapsto e_{2}+\beta, 0,-e_{2}+\beta, e_{2}+\beta,-e_{2}+\beta$, for $g_{1}=a, b, a^{2}, a b, a^{2} b$, and where $\Theta_{4}\left(1 \otimes g_{2} \otimes g_{3} \otimes g_{4} \otimes g_{5}\right)=-e_{1}$,

$1 \otimes g_{1} \otimes g_{2} \otimes g_{3} \otimes g_{4} \otimes g_{5} \mapsto 0$, for any other case,

$$
\Theta_{6}: A \otimes \bar{A}^{\otimes 6} \longrightarrow A e_{2}
$$

$1 \otimes g_{1} \otimes g_{2} \otimes g_{3} \otimes g_{4} \otimes g_{5} \otimes g_{6} \mapsto e_{2}, 0,0,0,-e_{2}$ for $g_{1}=a, b, a^{2}, a b, a^{2} b$,

and where $\Theta_{5}\left(1 \otimes g_{2} \otimes g_{3} \otimes g_{4} \otimes g_{5} \otimes g_{6}\right)=e_{2}+\beta$,

$1 \otimes g_{1} \otimes g_{2} \otimes g_{3} \otimes g_{4} \otimes g_{5} \otimes g_{6} \mapsto 0,0, e_{2},-e_{2}, 0$ for $g_{1}=a, b, a^{2}, a b, a^{2} b$, and where $\Theta_{5}\left(1 \otimes g_{2} \otimes g_{3} \otimes g_{4} \otimes g_{5} \otimes g_{6}\right)=e_{2}-\beta$,

$1 \otimes g_{1} \otimes g_{2} \otimes g_{3} \otimes g_{4} \otimes g_{5} \otimes g_{6} \mapsto-e_{2}, 0,0,0, e_{2}$ for $g_{1}=a, b, a^{2}, a b, a^{2} b$, and where $\Theta_{5}\left(1 \otimes g_{2} \otimes g_{3} \otimes g_{4} \otimes g_{5} \otimes g_{6}\right)=-e_{2}-\beta$,

$1 \otimes g_{1} \otimes g_{2} \otimes g_{3} \otimes g_{4} \otimes g_{5} \otimes g_{6} \mapsto 0,0,-e_{2}, e_{2}, 0$ for $g_{1}=a, b, a^{2}, a b, a^{2} b$, and where $\Theta_{5}\left(1 \otimes g_{2} \otimes g_{3} \otimes g_{4} \otimes g_{5} \otimes g_{6}\right)=-e_{2}+\beta$,

$1 \otimes g_{1} \otimes g_{2} \otimes g_{3} \otimes g_{4} \otimes g_{5} \otimes g_{6} \mapsto 0$, for any other case,

$$
\Theta_{7}: A \otimes \bar{A}^{\otimes 7} \longrightarrow A e_{1}
$$

$1 \otimes g_{1} \otimes g_{2} \otimes g_{3} \otimes g_{4} \otimes g_{5} \otimes g_{6} \otimes g_{7} \mapsto-e_{1}-\alpha, 0, e_{1}-\alpha, e_{1}+\alpha,-e_{1}+\alpha$ for $g_{1}=a, b, a^{2}, a b, a^{2} b$, and where $\Theta_{6}\left(1 \otimes g_{2} \otimes g_{3} \otimes g_{4} \otimes g_{5} \otimes g_{6} \otimes g_{7}\right)=e_{2}$,

$1 \otimes g_{1} \otimes g_{2} \otimes g_{3} \otimes g_{4} \otimes g_{5} \otimes g_{6} \otimes g_{7} \mapsto e_{1}+\alpha, 0,-e_{1}+\alpha,-e_{1}-\alpha, e_{1}-\alpha$ for $g_{1}=a, b, a^{2}, a b, a^{2} b$, and where $\Theta_{6}\left(1 \otimes g_{2} \otimes g_{3} \otimes g_{4} \otimes g_{5} \otimes g_{6} \otimes g_{7}\right)=-e_{2}$,

$1 \otimes g_{1} \otimes g_{2} \otimes g_{3} \otimes g_{4} \otimes g_{5} \otimes g_{6} \otimes g_{7} \mapsto 0$, for any other case,

$$
\Theta_{8}: A \otimes \bar{A}^{\otimes 8} \longrightarrow A e_{1} \text {, }
$$

$1 \otimes g_{1} \otimes g_{2} \otimes g_{3} \otimes g_{4} \otimes g_{5} \otimes g_{6} \otimes g_{7} \otimes g_{8} \mapsto e_{1}, 0,0,0, e_{1}$ for $g_{1}=a, b, a^{2}, a b, a^{2} b$, and where $\Theta_{7}\left(1 \otimes g_{2} \otimes g_{3} \otimes g_{4} \otimes g_{5} \otimes g_{6} \otimes g_{7} \otimes g_{8}\right)=e_{1}+\alpha$,

$1 \otimes g_{1} \otimes g_{2} \otimes g_{3} \otimes g_{4} \otimes g_{5} \otimes g_{6} \otimes g_{7} \otimes g_{8} \mapsto 0,0, e_{1}, e_{1}, 0$ for $g_{1}=a, b, a^{2}, a b, a^{2} b$, 
and where $\Theta_{7}\left(1 \otimes g_{2} \otimes g_{3} \otimes g_{4} \otimes g_{5} \otimes g_{6} \otimes g_{7} \otimes g_{8}\right)=e_{1}-\alpha$,

$1 \otimes g_{1} \otimes g_{2} \otimes g_{3} \otimes g_{4} \otimes g_{5} \otimes g_{6} \otimes g_{7} \otimes g_{8} \mapsto-e_{1}, 0,0,0,-e_{1}$ for $g_{1}=a, b, a^{2}, a b, a^{2} b$,

and where $\Theta_{7}\left(1 \otimes g_{2} \otimes g_{3} \otimes g_{4} \otimes g_{5} \otimes g_{6} \otimes g_{7} \otimes g_{8}\right)=-e_{1}-\alpha$,

$1 \otimes g_{1} \otimes g_{2} \otimes g_{3} \otimes g_{4} \otimes g_{5} \otimes g_{6} \otimes g_{7} \otimes g_{8} \mapsto 0,0,-e_{1},-e_{1}, 0$ for $g_{1}=a, b, a^{2}, a b, a^{2} b$,

and where $\Theta_{7}\left(1 \otimes g_{2} \otimes g_{3} \otimes g_{4} \otimes g_{5} \otimes g_{6} \otimes g_{7} \otimes g_{8}\right)=-e_{1}+\alpha$,

$1 \otimes g_{1} \otimes g_{2} \otimes g_{3} \otimes g_{4} \otimes g_{5} \otimes g_{6} \otimes g_{7} \otimes g_{8} \mapsto 0$, for any other case.

Note that both the representatives of $u$ and $v$ in the group cohomology $H^{*}\left(S_{3}\right)=\mathbb{F}_{3}[u, v] /\left(u^{2}\right)$ can be chosen as $A e_{1} \longrightarrow \mathbb{F}_{3}, e_{1} \mapsto 1, \alpha \mapsto 0, \beta \alpha \mapsto 0$. Since $|u|=3$ and $|v|=4$, we have $\widehat{\triangle}_{1}(u)=0$ and $\widehat{\triangle}_{1}(v)=\lambda u$ for some $\lambda \in \mathbb{F}_{3}$. We have

$$
\widehat{\triangle}_{1}(v)=\widehat{\triangle}_{1}\left(v \Theta_{4}\right) \circ \Lambda_{3},
$$

and $\widehat{\triangle}_{1}\left(v \Theta_{4}\right)$ can be computed by our formula in Theorem 8.2 . Since

$$
\begin{aligned}
& \widehat{\triangle}_{1}\left(v \Theta_{4}\right) \circ \Lambda_{3}\left(e_{1}\right)=e_{1} \widehat{\triangle}_{1}\left(v \Theta_{4}\right)(\beta \otimes \alpha \beta \otimes \alpha) \\
&=-\widehat{\triangle}_{1}\left(v \Theta_{4}\right)(\beta \otimes \alpha \beta \otimes \alpha)-b \widehat{\triangle}_{1}\left(v \Theta_{4}\right)(\beta \otimes \alpha \beta \otimes \alpha) \\
&=-2 \widehat{\triangle}_{1}\left(v \Theta_{4}\right)(\beta \otimes \alpha \beta \otimes \alpha)=\widehat{\triangle}_{1}\left(v \Theta_{4}\right)(\beta \otimes \alpha \beta \otimes \alpha) .
\end{aligned}
$$

By a MAPLE calculation (see [13]: A MAPLE program for computing $\widehat{\triangle}_{1}$.), we obtain that $\widehat{\triangle}_{1}\left(v \Theta_{4}\right)(\beta \otimes$ $\alpha \beta \otimes \alpha)=0$, and therefore $\widehat{\triangle}_{1}(v)=0$. Since $|u v|=7$ and $\left|v^{2}\right|=8$, we have $\widehat{\triangle}_{1}(u v)=\mu u^{2}$ and $\widehat{\triangle}_{1}\left(v^{2}\right)=\mu^{\prime} u v$ for some $\mu, \mu^{\prime} \in \mathbb{F}_{3}$. Since $u^{2}=0, \widehat{\triangle}_{1}(u v)=0$, and we only need to compute $\widehat{\triangle}_{1}\left(v^{2}\right)$. The representative of $v^{2}$ in the group cohomology $H^{*}\left(S_{3}\right)=\mathbb{F}_{3}[u, v] /\left(u^{2}\right)$ can also be chosen (up to a sign) as $A e_{1} \longrightarrow \mathbb{F}_{3}, e_{1} \mapsto 1, \alpha \mapsto 0, \beta \alpha \mapsto 0$. We have

$$
\begin{gathered}
\widehat{\triangle}_{1}\left(v^{2}\right)=\widehat{\triangle}_{1}\left(v^{2} \Theta_{8}\right) \circ \Lambda_{7}, \\
\widehat{\triangle}_{1}\left(v^{2} \Theta_{8}\right) \circ \Lambda_{7}\left(e_{1}\right)=e_{1} \widehat{\triangle}_{1}\left(v^{2} \Theta_{7}\right)(\beta \otimes \alpha \beta \otimes \alpha \otimes \beta \alpha \otimes \beta \otimes \alpha \beta \otimes \alpha) \\
=\widehat{\triangle}_{1}\left(v^{2} \Theta_{7}\right)(\beta \otimes \alpha \beta \otimes \alpha \otimes \beta \alpha \otimes \beta \otimes \alpha \beta \otimes \alpha) .
\end{gathered}
$$

Similarly by a MAPLE calculation (see[13]: A MAPLE program for computing $\widehat{\triangle}_{1}$ ), we obtain that $\widehat{\triangle}_{1}\left(v^{2} \Theta_{8}\right) \circ \Lambda_{7}\left(e_{1}\right)=0$, and therefore $\widehat{\triangle}_{1}\left(v^{2}\right)=0$.

Finally, based on the above computations, we deal with the Lie brackets. Since we have the following Possion rule: $[\alpha \cup \beta, \gamma]=[\alpha, \gamma] \cup \beta+(-1)^{|\alpha|(|\gamma|-1)} \alpha \cup[\beta, \gamma]$, it suffices to write down the Lie brackets between generators in $H H^{*}\left(\mathbb{F}_{3} S_{3}\right)$. Recall that $H H^{*}\left(\mathbb{F}_{3} S_{3}\right)$ is generated as an algebra by elements $u, v, C_{1}=1+a+a^{2}, C_{2}=b\left(1+a+a^{2}\right), X_{1}, X_{2}$ of degrees (resp.) 3, 4,0,0,1 and 2 , subject to the relations

$$
\begin{gathered}
u X_{1}=0, \quad v X_{1}=u X_{2}, \quad u C_{2}=0=v C_{2}, \\
C_{i} X_{j}=0=C_{i} C_{j}(i, j \in\{1,2\}), \quad X_{1} X_{2}=u C_{1}, \quad X_{2}^{2}=v C_{1}
\end{gathered}
$$

in addition to the graded commutative relations. Using the formulas (Here we omit the sign $\cup$ in the equation)

and

$$
[\alpha, \beta]=-(-1)^{(|\alpha|-1)|\beta|}\left(\triangle(\alpha \beta)-\triangle(\alpha) \beta-(-1)^{|\alpha|} \alpha \triangle(\beta)\right)
$$

we do the concrete computations as follows:

$$
[\alpha, \beta]=-(-1)^{(|\alpha|-1)(|\beta|-1)}[\beta, \alpha],
$$

$$
\begin{gathered}
{[u, u]=0,[u, v]=\widehat{\triangle}_{1}(u v)-\widehat{\triangle}_{1}(u) v+u \widehat{\triangle}_{1}(v)=0,[v, u]=0,} \\
{\left[u, C_{1}\right]=-\left(\triangle\left(u C_{1}\right)-\triangle(u) C_{1}+u \triangle\left(C_{1}\right)\right)=-\widehat{\triangle}_{a}\left(X_{1} X_{2}\right)=X_{2},\left[C_{1}, u\right]=-X_{2},} \\
{\left[u, C_{2}\right]=-\left(\triangle\left(u C_{2}\right)-\triangle(u) C_{2}+u \triangle\left(C_{2}\right)\right)=0,\left[C_{2}, u\right]=0,} \\
{\left[u, X_{1}\right]=-\left(\triangle\left(u X_{1}\right)-\triangle(u) X_{1}+u \triangle\left(X_{1}\right)\right)=u,\left[X_{1}, u\right]=-u,} \\
{\left[u, X_{2}\right]=-\left(\triangle\left(u X_{2}\right)-\triangle(u) X_{2}+u \triangle\left(X_{2}\right)\right)=-\widehat{\triangle}\left(u X_{2}\right)=0^{c},\left[X_{2}, u\right]=0,} \\
{[v, v]=-\left(\triangle\left(v^{2}\right)-\triangle(v) v-v \triangle(v)\right)=0,} \\
{\left[v, C_{1}\right]=-\left(\triangle\left(v C_{1}\right)-\triangle(v) C_{1}+v \triangle\left(C_{1}\right)\right)=-\widehat{\triangle}_{a}\left(X_{2}^{2}\right)=0,\left[C_{1}, v\right]=0,}
\end{gathered}
$$

\footnotetext{
${ }^{\mathrm{c}} u X_{2}$ is an element of degree 5, under the additive decompositon, it correponds to an element in $H^{*}(\langle a\rangle)$ and has the form $\pm w_{1} w_{2}{ }^{2}$. It follows from the formula in the last paragragh of Section 8 that $\widehat{\triangle}\left(u X_{2}\right)=\widehat{\triangle}_{a}\left( \pm w_{1} w_{2}{ }^{2}\right)=0$.
} 


$$
\begin{gathered}
{\left[v, C_{2}\right]=-\left(\triangle\left(v C_{2}\right)-\triangle(v) C_{2}+v \triangle\left(C_{2}\right)\right)=0,\left[C_{2}, v\right]=0,} \\
{\left[v, X_{1}\right]=\triangle\left(v X_{1}\right)-\triangle(v) X_{1}-v \triangle\left(X_{1}\right)=-v,\left[X_{1}, v\right]=v,} \\
{\left[v, X_{2}\right]=-\left(\triangle\left(v X_{2}\right)-\triangle(v) X_{2}-v \triangle\left(X_{2}\right)\right)= \pm \widehat{\triangle}_{a}\left(X_{2}^{3}\right)=0,\left[X_{2}, v\right]=0,} \\
{\left[C_{1}, C_{1}\right]=\left[C_{1}, C_{2}\right]=\left[C_{2}, C_{1}\right]=\left[C_{2}, C_{2}\right]=0,} \\
{\left[C_{1}, X_{1}\right]=\triangle\left(C_{1} X_{1}\right)-\triangle\left(C_{1}\right) X_{1}-C_{1} \triangle\left(X_{1}\right)=C_{1},\left[X_{1}, C_{1}\right]=-C_{1},} \\
{\left[C_{1}, X_{2}\right]=-\left(\triangle\left(C_{1} X_{2}\right)-\triangle\left(C_{1}\right) X_{2}-C_{1} \triangle\left(X_{2}\right)\right)=0,\left[X_{2}, C_{1}\right]=0,} \\
{\left[C_{2}, X_{1}\right]=\triangle\left(C_{2} X_{1}\right)-\triangle\left(C_{2}\right) X_{1}-C_{2} \triangle\left(X_{1}\right)=C_{2},\left[X_{1}, C_{2}\right]=-C_{2},} \\
{\left[C_{2}, X_{2}\right]=-\left(\triangle\left(C_{2} X_{2}\right)-\triangle\left(C_{2}\right) X_{2}-C_{2} \triangle\left(X_{2}\right)\right)=0,\left[X_{2}, C_{2}\right]=0,} \\
{\left[X_{1}, X_{1}\right]=0,\left[X_{1}, X_{2}\right]=-\left(\triangle\left(X_{1} X_{2}\right)-\triangle\left(X_{1}\right) X_{2}+X_{1} \triangle\left(X_{2}\right)\right)=0,\left[X_{1}, X_{2}\right]=0,} \\
{\left[X_{2}, X_{2}\right]=-\left(\triangle\left(X_{2}^{2}\right)-\triangle\left(X_{2}\right) X_{2}-X_{2} \triangle\left(X_{2}\right)\right)=0 .}
\end{gathered}
$$

Remark 9.1. By a recent result of Menichi (see [15, p. 321]), the Lie bracket of the group cohomology $H^{*}(G)$ for a finite group $G$ must be trivial. The above computation shows that this is indeed the case for $H^{*}\left(S_{3}\right)=\mathbb{F}_{3}[u, v] /\left(u^{2}\right)$. Note that to verify $[v, v]=0$, we have used the MAPLE program in 13 .

Remark 9.2. Observe in the above example that the generators of $H H^{*}\left(\mathbb{F}_{3} S_{3}\right)$ are "multiplicative closed" under Lie bracket: the Lie bracket $[\alpha, \beta]$ of two generators $\alpha$ and $\beta$ is a scalar multiple of another generator. Also if $[\alpha, \beta] \neq 0$, then $[\alpha, \beta]$ is equal to $-[\beta, \alpha]$.

\section{REFERENCES}

[1] N.Bian, P.Zhang and G.Zhang, Setwise homotopy category. Appl. Categ. Structures 17(6) (2009), 561-565.

[2] D.Benson, Representations and cohomology II: Cohomology of groups and modules. Cambridge University Press, 1991.

[3] C.Cibils, Tensor product of Hopf bimodules over a group. Proc. Amer. Math. Soc. 125 (1997), 1315-1321.

[4] C.Cibils and A.Solotar, Hochschild cohomology algebra of abelian groups. Arch. Math. 68 (1997), 17-21.

[5] S.Eilenberg and S.Mac Lane, Cohomology theory in abstract groups I. Ann. Math. 48 (1947), 51-78.

[6] L.Evens, The cohomology of groups. Clarendon Press, 1991.

[7] M.A.Farinati and A.L.Solotar, G-structure on the cohomology of Hopf algebras. Proc. Amer. Math. Soc. 132 (2004), 2859-2865.

[8] M.Gerstenhaber, The cohomology structure of an associative ring. Ann. Math. 78(2) (1963), 267-288.

[9] E.Getzler, Batalin-Vilkovisky algebras and two-dimensional topological field theories. Comm. Math. Phys. 159(2) (1994), 265-285.

[10] T.Holm, The Hochschild cohomology ring of a modular group algebra: the commutative case. Comm. Algebra 24 (1996), 1957-1969.

[11] J.Le And G.D.Zhou, On the Hochschild cohomology of tensor product of algebras. To appear in J. Pure Appl. Algebra.

[12] J.L.Loday, Cyclic Homology. Grundlehren der mathematischen Wissenschaften Vol. 301, Springer, 1998.

[13] Y.M.Liu And G.D.Zhou, A Maple program, avalible at http://math.bnu.edu.cn/ liuym/

[14] L.Menichi, Batalin-Vilkovisky algebras and cyclic cohomology of Hopf algebras. K-Theory 32(3) (2004), $231-251$.

[15] L.Menichi, Connes-Moscovici characteristic map is a Lie algebra morphism. J. Algebra 331 (2011), 311-337.

[16] S.S.Siegel And S.J.Witherspoon, The Hochschild cohomology ring of a group algebra. Proc. London Math. Soc. 79(3) (1999), 131-157.

[17] R.TAlllefer, Injective Hopf bimodules, cohomologies of infinite dimensional Hopf algebras and gradedcommutativity of the Yoneda product. J. Algebra 276 (2004), 259-279.

[18] T.Tradler, The Batalin-Vilkovisky algebra on Hochschild cohomology induced by infinitely inner products. Ann. Inst. Fourier (Grenoble) 58(7) (2008), 2351-2379.

[19] T.YAng, A Batalin-Vilkovisky Algebra Structure on the Hochschild Cohomology of Truncated Polynomials. Topology and its Applications 160(13) (2013), 1633-1651.

YuMing LiU

School of Mathematical Sciences

Laboratory of Mathematics and Complex Systems

Beijing Normal University

Beijing 100875

P.R.CHINA

E-mail address: ymliu@bnu.edu.cn 
GUODONG ZHOU

Department of Mathematics

Shanghai Key Laboratory OF PMMP

East China Normal University

Dong Chuan Road 500

SHANGHAi 200241

P.R.CHINA

E-mail address: gdzhou@math.ecnu.edu.cn 